\title{
基于深度学习的三维点云修复技术综述
}

\author{
刘彩霞 ${ }^{1)}$, 魏明强 ${ }^{2}{ }^{*}$, 郭延文 ${ }^{3)}$ \\ 1) (南京师范大学教育信息工程研究所 南京 210097) \\ 2) (南京航空航天大学计算机科学与技术学院 南京 211106) \\ 3) (南京大学计算机软件新技术国家重点实验室 南京 210023) \\ (mqwei@nuaa.edu.cn)
}

\begin{abstract}
摘 要: 三维点云是最常用的三维场景/物体表示方法之一. 根据点云修复侧重点不同, 将基于深度学习的三维点云 修复技术划分为密集重建、补全重建和去噪重建 3 类; 详细分析了相关典型修复模型及关键技术，如特征编码、特征 扩展和损失函数设计; 总结了常用的网络模块、点云数据集和评估准则; 最后讨论了 3 类修复技术之间的关系，并从 旋转不变性特征提取、细节信息修复、拓扑关系保持、几何算法应用和多模态数据融合 5 个方面探讨了点云修复技 术面临的挑战及未来发展趋势.
\end{abstract}

关键词：三维点云；点云修复；深度学习; 网络模型；特征编码

中图法分类号: TP391.41 DOI: 10.3724/SP.J.1089.2021.18817

\section{D Point Cloud Restoration via Deep Learning: A Comprehensive Survey}

\author{
Liu Caixia ${ }^{1)}$, Wei Mingqiang ${ }^{2)^{*}}$, and Guo Yanwen ${ }^{3)}$ \\ 1) (Institute of EduInfo Science \& Engineering, Nanjing Normal University, Nanjing 210097) \\ 2) (College of Computer Science and Technology, Nanjing University of Aeronautics and Astronautics, Nanjing 211106) \\ 3) (State Key Laboratory for Novel Software Technology, Nanjing University, Nanjing 210023)
}

\begin{abstract}
D point cloud is one of the most commonly used 3D scene/object representation methods. According to the different emphases of point cloud restoration, 3D point cloud restoration technologies based on deep learning are divided into three classes: dense reconstruction, complete reconstruction and denoising reconstruction. Typical restoration models and key techniques, such as feature coding, feature extension, and loss function design, are analyzed. Commonly used network modules, point cloud data sets, and evaluation criteria are summarized. Finally, the relationship between the three kinds of point cloud restoration technologies is discussed, and the challenges and future development trends of point cloud restoration technologies are explored from five aspects: rotation invariant feature extraction, detail information repair, topological relationship preservation, geometric algorithm application, and multimodal data fusion.
\end{abstract}

Key words: 3D point cloud; point cloud restoration; deep learning; network module; feature coding

三维点云是三维坐标系统中具有无序性、无连 接性的一组空间坐标点的集合. 与多边形网格曲 面相比, 点云不需要存储和维护网格的连接性 ${ }^{[1]}$ 或
拓扑一致性 ${ }^{[2]}$. 由于其具有简单、灵活和强大的表 示能力 ${ }^{[3-5]}$, 因此其在文物修复、自动驾驶和机器 人等诸多研究领域得到了广泛的使用 ${ }^{[6-8]}$.

收稿日期: 2020-11-28; 修回日期: 2021-02-09. 基金项目: 国家自然科学基金(62032011, 62172218,62007028); 江苏省文化和旅 游科研课题项目(20ZD06); 中央引导地方科技发展基金自由探索类基础研究项目(2021Szvup060). 刘彩霞(1980一), 女, 博士研究生, 主要研究方向为数字图像处理、计算机图形学; 魏明强(1985一), 男, 博士, 副教授, CCF 会员, 论文通讯作者, 主要研究方向为计算 机图形学; 郭延文(1981一), 男, 博士, 教授, CCF 会员, 主要研究方向为计算机图形学、多媒体处理. 
点云数据可以由三维扫描设备产生, 如激光 雷达、立体摄像头、Kinect 和 ToF(time-of-flight) 相机等. 然而, 受采集设备固有的误差、场景物体 表面的照明或反射属性等因素的影响, 传感器采集 的点云不可避免地包含噪声和异常值 ${ }^{[9]}$. 与此同时, 采集设备有限的分辨率、硬件和计算能力的限制以 及遮挡等因素常常导致数据点稀疏甚至缺失 ${ }^{[10-11]}$, 严重影响后续的曲面重建和点云分类. 因此, 有必 要对原始点云进行修复操作.

设 $X$ 为一组三维空间点, 且这些点位于由一 个三维传感器的单次观测或一系列观测得到的物 体观察表面上, $Y$ 为从该物体表面均匀采样得到的 三维点集真值. 这里, $X$ 不一定是 $Y$ 的子集, 并且 由于 $X$ 中的点和 $Y$ 中的点都独立于物体基本表面, 因此它们之间没有明确的对应关系．点云修复问 题可以定义为根据给定的 $X$ 预测 $Y$, 其目的是通过 对点云密度的提升、形状的补全以及噪声的去除等 提高点云质量. 即对于给定点云数据集, 从知识库 中获取先验特征或学习其潜在特征, 对所选择的 特征进行适当的变形和重构, 重建保持几何细节 的、密集的、均匀的、完整的和干净的点云模型. 传 统的点云分析和处理主要建立在手工定义的模型 特征之上，只能针对特定的问题或满足特定的条 件才能有效. 而深度学习在自然语言和图像处理 方面的成功应用，展示了其作为数据特征提取工 具的强大能力. 相关深度学习技术在三维点云处 理中也得到了广泛应用. PointNet ${ }^{[12]}$ 及其扩展 PointNet $+{ }^{[13]}$ 是这一领域的先驱, 实现了端对端 的三维数据特征自动提取. 同时, 它们将多层感知 器与对称聚合函数相结合, 实现了输人排列的不 变性和扰动的鲁棒性. PointNet 和 PointNet++的出 现, 极大地促进了基于深度学习的三维点云修复 技术的发展.

本文立足于三维点云修复问题，对近年来基 于深度学习的点云上采样、形状补全和点云去噪模 型从技术层面进行了梳理和分析.

\section{1 相关基础网络模型}

基于深度学习的点云修复中常用的基础网络 模型包括多层感知器(multilayer perceptron, MLP)、 卷积神经网络 (convolutional neural networks, $\mathrm{CNN}$ )、自编码器(autoencoder, AE) 和对抗生成网络 (generative adversarial networks, GAN)等.

\subsection{MLP}

MLP 是一类前馈人工神经网络，通常由一个 输人层、一个或多个隐藏层和一个输出层组成, 如 图 1 所示. 其中, $L_{1} \sim L_{3}$ 分别为输人层、隐藏层和 输出层, $x_{i}(i=1,2,3)$ 为输人数据, $a_{i}^{(2)}(i=1,2,3)$ 为 $L_{2}$ 层的输出数据. 由于 MLP 是完全连接的, 所 以一层中的每个节点都用一定的权值连接到下一 层中的每个节点. MLP 使用反向传播的监督学习 技术进行训练, 根据输出与预期结果之间的误差 值改变连接权值来学习特征和目标之间的关系. MLP 应用范围较广, 扩展性也强, 并且能够处理 非线性可分离的问题.

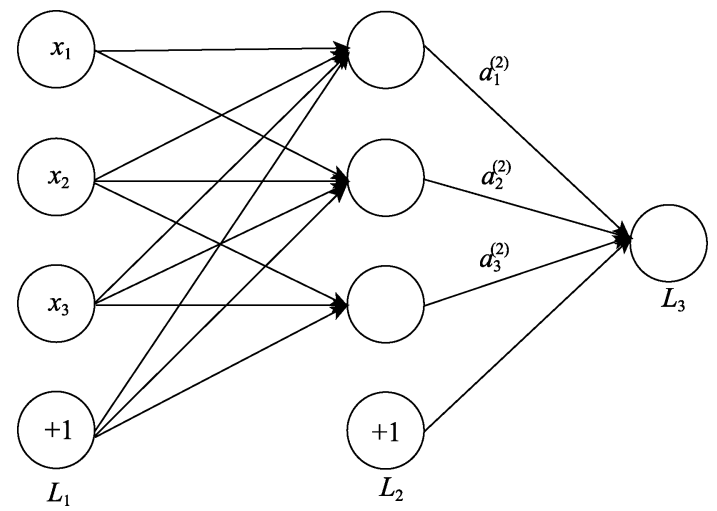

图 $1 \mathrm{MLP}$

在 MLP 中，通常使用一个非线性可微的激活 函数来模拟生物神经元的动作电位或放电频率. 常见的激活函数包括 Sigmoid 函数 $\left(f(x)=\frac{1}{1+\mathrm{e}^{-x}}\right)$ 和双曲正切函数 $\left(\tanh (x)=\frac{\mathrm{e}^{x}-\mathrm{e}^{-x}}{\mathrm{e}^{x}+\mathrm{e}^{-x}}\right)$ 等.

\section{$1.2 \mathrm{CNN}$}

CNN 通常由输人层、卷积层、激活函数、池 化层、全连接层等组成，如图 2 所示.

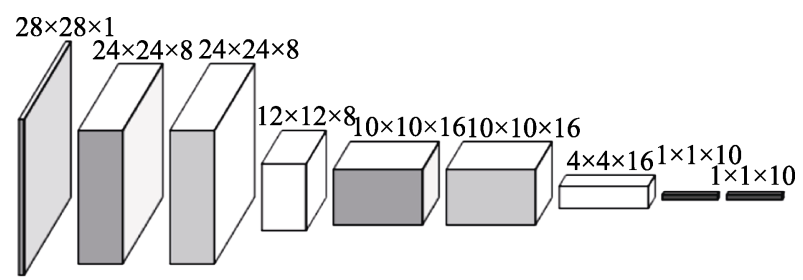

图 $2 \mathrm{CNN}$

其中, 卷积层是一组平行的特征图, 特征图中 的元素通过在输人图像上滑动卷积核并进行相应 的计算得出, 即一幅特征图共享了相同的权重和 
偏置项. 池化层一般位于卷积层之后, 是一种非线 性形式的降采样, 可以不断地减小数据的空间大 小, 从而降低参数的数量和计算量, 在一定程度上 达到控制过拟合的目的. 全连接层负责完成神经 网络中的高级推理. 最后, 使用损失函数评价模型 的预测值和真实值相异的程度. 此外, 在 CNN 中, 卷积层后面常常伴随激励函数, 它可以增强判定 函数和整个神经网络的非线性特性，常用的激励 函数包括修正线性单元 (rectified linear units, ReLU)、双曲正切函数和 Sigmoid 函数等.

CNN 通过局部感受野、共享权值和空间子采 样等操作实现了一定程度的平移和变形不变性, 共享权值的使用也减少了系统辅助泛化中参数的 数量 ${ }^{[14]}$, 因此 CNN 成为深度学习中常用的基础网 络模型.

\section{$1.3 \mathrm{AE}$}

$\mathrm{AE}^{[15-16]}$ 属于无监督机器学习算法, 如图 3 所 示. 其目的是对一组数据学习出一种表示(也称为 表征或编码), 然后通过这种表征来重构输出. AE 通常由编码器和解码器 2 部分组成. 编码器负责将 输人压缩成潜在空间表征, 解码器负责重构来自 潜在空间表征的输人. $\mathrm{AE}$ 具有数据相关性、数据有 损性和自动学习性等特点, 常常用于数据的去噪 和降维等.

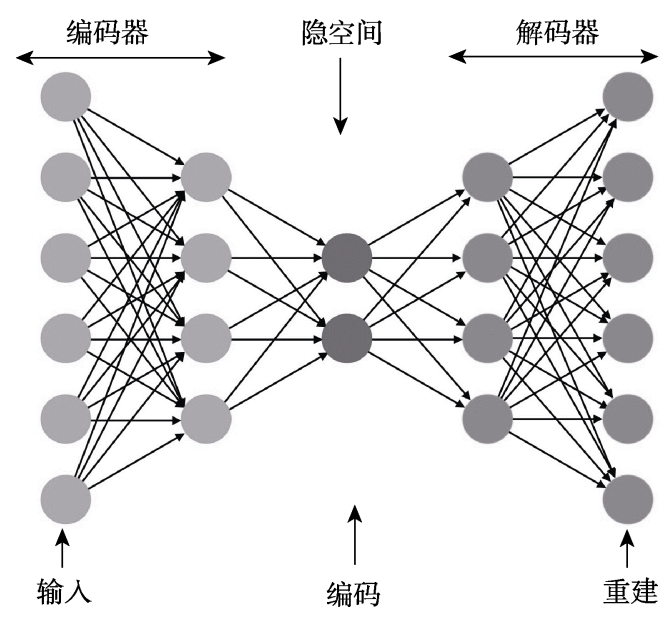

图 $3 \mathrm{AE}$

\subsection{GAN}

$\mathrm{GAN}^{[17]}$ 是一种无监督式学习网络, 通过让生 成神经网络和判别神经网络相互博弯的方式进行 学习, 如图 4 所示. 生成网络从潜在空间中随机取 样作为输人, 其输出结果需要尽量模仿训练集中 的真实样本. 判别网络的输人为真实样本或生成 网络的输出, 其目的是尽可能从真实样本中分辨
出生成网络输出结果. 而生成网络要尽可能地欺 骗判别网络. 2 个网络相互对抗, 不断调整参数, 最终使判别网络无法判断生成网络的输出结果是 否真实.

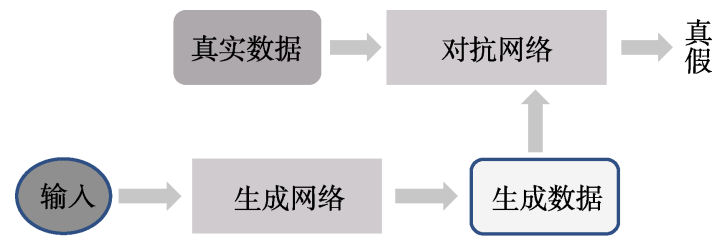

图 4 GAN

\section{2 三维点云修复技术}

本节先对点云修复技术进行分类，然后分别 介绍并分析 3 类基于深度学习的点云修复技术.

\section{1 点云修复分类}

根据修复任务的侧重点不同，可以将基于深 度学习的点云修复技术划分为密集重建、补全重建 和去噪重建 3 类, 如图 5 所示 ${ }^{[10,18-19]}$.

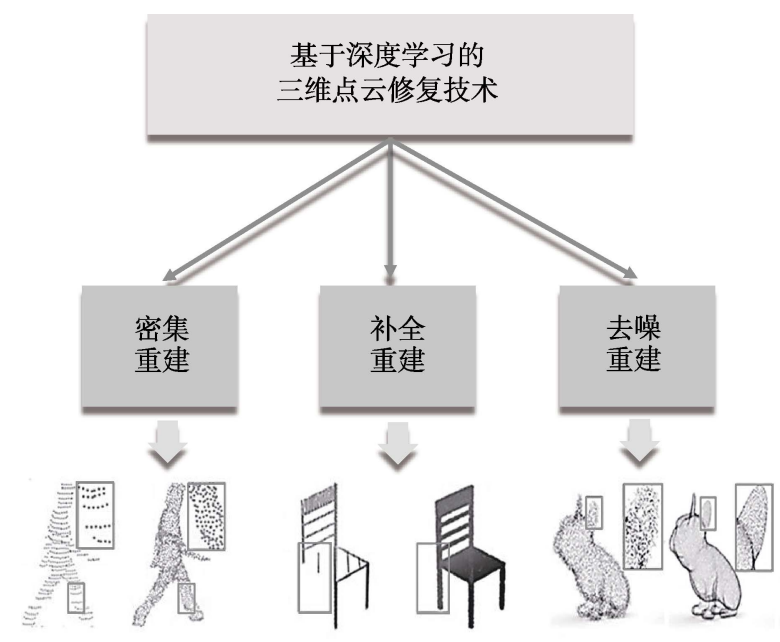

图 5 三维点云重建

(1) 侧重于提升局部点云块内部点的分辨率 (密度)和分布均匀性的修复操作, 称之为密集重建 (上采样重建或超分辨率重建);

(2) 聚焦于三维形状补全的点云修复操作，称 之为补全重建;

（3）偏重于保留底层对象表面的几何细节的 同时从噪声输人中恢复一个干净点集的操作, 称 之为去噪重建.

\section{2 密集重建}

点云表示几何形状(通常是它的表面), 每个点 至少包含欧几里得空间中的一组三维坐标 $(x, y, z)$. 
因此，一个物体或场景的点云可以简单表示为一 个 $N \times 3$ 的矩阵. 其中 $N$ 为点的个数, 称为点云分 辨率. 由深度相机和激光雷达传感器产生的原始 点云通常是低分辨率或稀疏的，同时点云分布也 是不均匀的. 密集重建的目标是通过给定的一组 非均匀分布的、稀疏的 $N \times 3$ 点云坐标，在保持目标 对象形状(潜在表面)的前提下，输出一个密度更大 的、分布均匀的 $r N \times 3$ 点云. 其中, $r$ 为上采样率.

基于 PointNet++体系结构, Yu 等 ${ }^{[20]}$ 首先将深 度神经网络引人点云密集重建任务中, 提出如图 6 所示 PU-Net 网络架构, 其主要包括点云块提取、 点特征嵌人、特征扩展和坐标重建 4 个部分. 首先, 从一组已知的三维模型中通过下采样提取不同尺 度和分布的点云块; 然后, 通过层次特征学习和多 级特征聚合将原始三维坐标映射到特征空间; 最 后, 将扩充后的特征进行聚合, 并通过一组全连接 层重建为上采样点云坐标. 在训练过程中, 利用联
合损失函数约束新生成点的位置和分布. 但是, 由 于点集没有规则的结构, 点的邻域也不是固定的, 因此邻域信息一般通过 $K$ 近邻搜索收集, 而这种 逐层逐点的计算是相当耗时的. 同时, 下采样操作 也容易丢失几何细节信息 ${ }^{[18]}$. 与 PU-Net 网络结构 相似, Wang 等 ${ }^{[21]}$ 提出多步递进的上采样网络, 也 称为 3PU(3-step progressive upsampling), 其在多 个步骤中学习不同级别的细节. 该网络模型随机 选取一个局部点云块作为输人, 在真值的指导下 对点云块进行采样, 并将结果传递到下一步. 通过 逐步地训练基于点云块的端对端网络, 将稀疏的 输人点集逐步重建为一个具有丰富几何细节的密 集点集. 但是, 基于点云块操作的密集重建结果往 往缺乏精细的几何结构. Zhang 等 ${ }^{[22]}$ 提出了一种数 据驱动的点云上采样技术, 将整个点云数据作为 密集重建网络的输人, 它能够学习不同类别的点 云的潜在特征，重建密集三维点云.

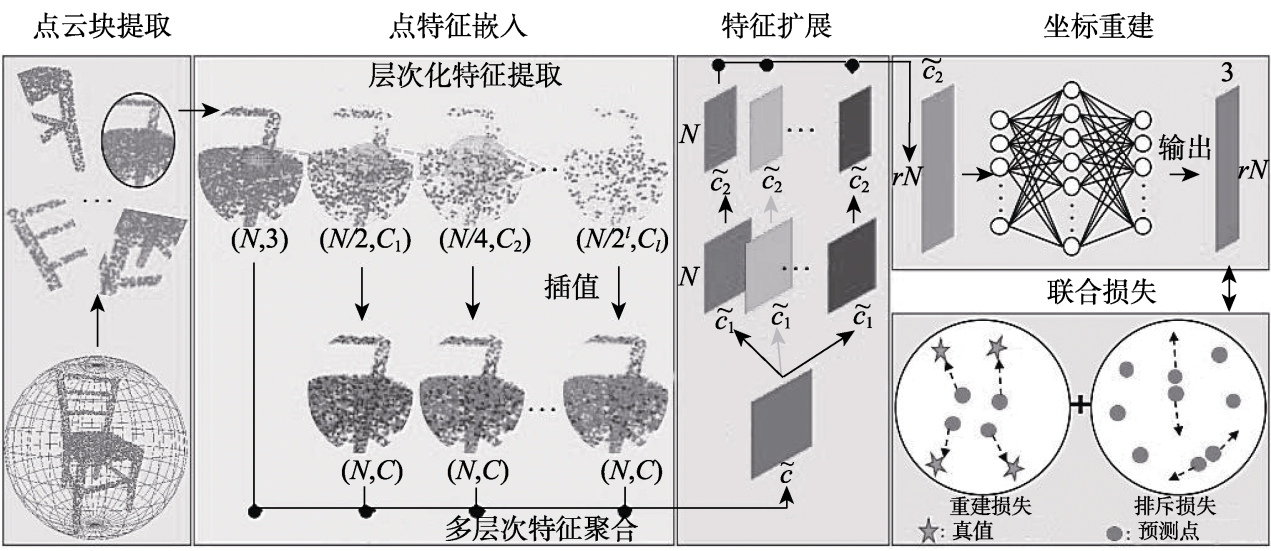

图 6 PU-Net 点云密集重建模型 ${ }^{[20]}$

图卷积网络(graph convolutional networks, GCN) 是一种能对图数据进行深度学习的技术 ${ }^{[23-24]}$. Qian 等 ${ }^{[11]}$ 通过融合 GCN 和 DenseGCN ${ }^{[25]}$ 的特征提取器 进行三维点云特征提取，并通过 Multi-branch GCN, Clone GCN, NodeShuffle 等 GCN 模块进行特征扩 展, 最后通过坐标重建得到密集的点云. 与传统的 CNN 相比, GAN 框架利用一个鉴别器隐式地学习 评价生成器生成的对象，这种灵活性特别有利于 生成性任务 ${ }^{[26-27]}$. $\mathrm{Li}$ 等 ${ }^{[18]}$ 的密集点云重建框架 PU-GAN 将上采样与数据修正相结合，利用对抗 网络训练发生器从潜在空间产生丰富多样的点分 布, 利用鉴别网络隐式地评估从发生器产生的点 集对潜在点分布的影响. PU-GAN 关注于提高上采 样点云的密度和均匀性，同时设计了特征提取模 块和特征扩展模块.

\section{3 补全重建}

在三维数据捕获过程中, 有限的传感器分辨 率和物体遮挡等原因常常导致采集到的数据点是 不完全的和稀疏的. 补全重建的任务就是从残缺 的或局部的输人数据中恢复一个完整的形状，其 在三维重建中有重要的价值.

形状补全操作需要满足以下 3 个要求 ${ }^{[28]}$ : (1) 保留输人点云 $X$ 的细节; (2) 用详细的几何结构绘 制缺失的部分; (3) 在物体表面生成均匀分布的点. 用于补全重建的神经网络结构通常由编码器和解 码器 2 部分构成. 编码器的功能是将输人的残缺点 云提取出形状特征 $\boldsymbol{G}$, 而解码器通常利用 $\boldsymbol{G}$ 并采用 一个由粗到细的策略恢复出完整的三维点云.

补全重建在一定程度上可以看作带有形状校 正的密集重建过程. Yuan 等 ${ }^{[10]}$ 提出的基于深度学 
习的点补全网络(point completion network, PCN), 如图 7 所示. PCN 直接对原始点云进行操作，没有 任何关于底层形状的结构假设(如对称性)或注释 (如语义类). 其网络模型包括编码器和解码器 2 部 分, 编码器使用基于 MLP 和最大池化操作的网络 模型提取输人点云的全局特征; 解码器分别利用 全连接层和折叠网络 ${ }^{[29]}$ 获得稀疏的点云坐标和平 滑的密集点云. 与 PCN 类似, Huang 等 ${ }^{[30]}$ 提出直接 作用于点云的多级神经网络结构, 将输人的残缺 点云重建成完整的点云. 由于输人点云形状不完 整，因此在利用 MLP 进行全局特征提取时容易丢 失局部细节信息. Zhang 等 ${ }^{[31]}$ 在 PCN 的特征编码器 中引人了一个注意力单元突出重要特征的权重, 然后使用基于多阶段 PCN 的多级架构解码器进行 点云重建，并利用基于不同分辨率的临界点集监 测不同的阶段. TopNet 模型 ${ }^{[32]}$ 使用了一个层次结 构的树状网络进行点云形状补全, 其不需要为输 人点集假设任何特定的拓扑结构. PF-Net 模型 ${ }^{[33]}$ 利用基于特征点的多尺度生成网络分层估计缺失 点云, 进一步将多阶段补全损失和对抗损失相加, 得到更符合实际情况的缺失区域. Chen 等 ${ }^{[28]}$ 设计
了一个基于 GAN 的点云补全网络, 其中, 生成器 负责将原始的部分点集映射为干净的和完整的点 集; 鉴别器用来鉴别生成数据集和真实数据. 类似 地, Wang 等 ${ }^{[34]}$ 通过扩展通用 GAN 框架, 将生成器 建模为特征提取编码器和带条件的由粗到精的解 码器, 而鉴别器用于区分生成器的输出结果是虚 假数据还是真实数据. 该模型同时考虑局部细节 和全局形状信息, 可以在不完整的点集中保留现 有的细节，同时补全缺失部分. Zhang 等 ${ }^{[35]}$ 提出了基 于分离特征聚合策略的补全重建算法, 将不同的特 征聚合起来分别表示已知部分和缺失部分, 并提出 了如图 8 所示 2 种不同的特征聚合策略, 即全局和局 部特征聚合以及残差特征聚合. 同样为了弥补全局 特征带来的细节损失问题, Wen 等 ${ }^{[36]}$ 提出了一种用 于三维点云补全的跳跃注意力网络, 主要包括用于 多层次特征提取的编码器、结构保持的解码器以及 跳跃注意力三大模块. 在使用 PointNet++提取的不 同层次特征的基础上, 跳跃注意力连接模块选择性 地将不完全点云的局部区域的几何信息传递给解码 器不同层次的折叠网络模块, 通过分层折叠网络逐 步细化局部区域来重建完整点云.

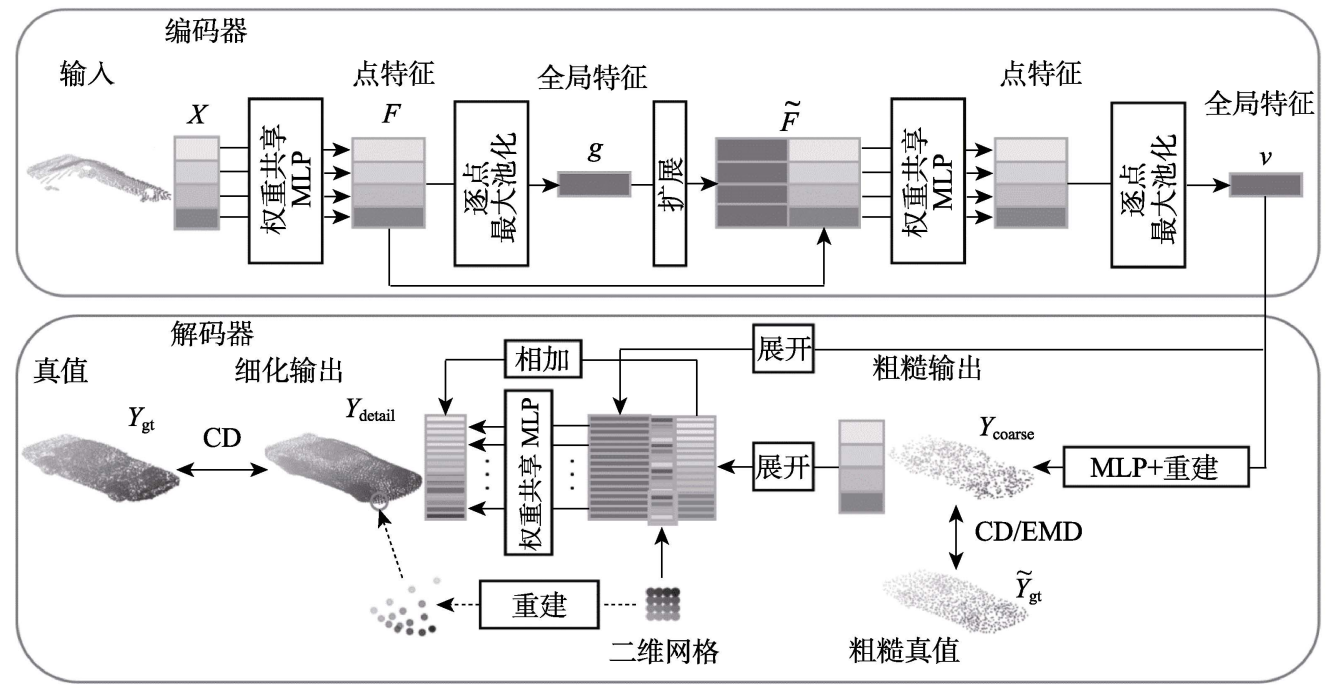

图 7 PCN 补全重建网络结构 ${ }^{[10]}$

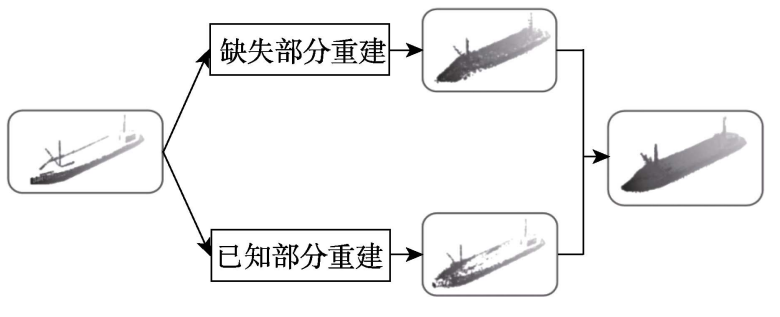

图 8 点云补全重建中的特征聚合策略 ${ }^{[35]}$

\section{4 去噪重建}

三维扫描设备(如深度相机和激光雷达传感
器)捕获的点云不可避免地包含噪声. 点云去噪的 目标是从噪声输人中恢复一个干净的点集, 同时 保留对象表面的几何细节. 实现这一目标的主要 技术挑战有 2 个方面 ${ }^{[19]}$.

（1）如何在不假设噪声分布的前提下适应各 种噪声;

（2）如何在去噪后的点集中保留几何细节(如 尖锐的边缘和光滑的表面).

GraphPointNet 模型 ${ }^{[37]}$ 将 GCN引人点云去噪中, 
利用边缘条件卷积 (edge-conditioned convolution, ECC $)^{[38]}$ 实现点云上的卷积运算. GraphPointNet 将 每个点云看作一个图，将点云中的每个点看做一 个节点，它们之间的加权连接作为边. ECC 作用在 一个深度神经网络中, 对第 $l+1$ 层的节点相关联的 特征向量进行计算, 并把它作为第 $l$ 层节点和其邻 近节点的特征向量加权局部聚合. Duan 等 ${ }^{[39]}$ 提出了 类似 PointNet 的神经网络构架进行三维点云去噪, 称为神经投影去噪模型. 它利用神经网络提取的点 云特征估计噪声点云的参考平面, 然后将噪声点投 影到相应的估计参考平面上, 对点云进行降噪处 理. 神经投影去噪模型可以去除点云噪声而无须根 据点云相邻点来估计参考平面, 也无须通过计算特 征分解来估计局部几何形状. 它可以利用局部和全 局信息进行法矢量估计, 受噪声强度和曲率变化的 影响较小. 在 PointNet 的基础上, Rakotosaona 等 ${ }^{[40]}$ 提出了深度学习网络框架 PointCleanNet 进行点云 去噪. 其去噪过程分为 2 个阶段: 第 1 阶段是离群 点去除, 预测点云中与每个点相关的离群点概率, 如果概率大于某一阈值, 则认为该点是离群点, 将 它从输人点云中删除; 第 2 阶段通过估计剩余点到 原始未知曲面的位移得到最终的去噪点云. EC-Net 模型 ${ }^{[41]}$ 中, 对于输人块(一组点云局部点)中的每个
点, 首先, 使用 PointNet++将其局部特征编码为向 量 $\boldsymbol{F}$; 然后, 使用特征扩展机制将 $\boldsymbol{F}$ 扩展为 $\boldsymbol{F}^{\prime}$, 在 此基础上，对 $\boldsymbol{F}^{\prime}$ 进行残差点坐标和点对边距离的回 归, 将原始点坐标与残差点坐标相加, 得到输出点 坐标; 最后, 识别边缘上的点并生成输出点云. Luo 等 ${ }^{[22]}$ 提出的三维点云去噪模型主要包括特征提取、 可微池化和流形重构等模块, 旨在通过从微噪声扰 动的下采样点及其嵌人的邻域特征学习有噪声点云 的底层流形, 以捕获点云的内在结构. 该模型以有 噪声的点云为输人, 通过可微的池化层对低噪声点 子集进行采样; 然后根据采样点的子集重建流形; 最后, 通过对重构流形采样得到去噪点云. 尽管一 些算法改进了去噪结果, 但它们的性能通常受到局 部接受域准确性的限制, 无法处理分散的点. 此外, 一些模型是局部的, 没有考虑三维对象和场景中固 有的非局部自相似. Huang 等 ${ }^{[19]}$ 引入了如图 9 所示 非局部感知(non-local part-aware, NLPA)网络结构, 从一个含有 $N$ 个三维笛卡尔系坐标点的噪声点云集 合上重建无噪声点集. 首先, 使用 MLP 将有噪声的 输人 $\mathcal{P}$ 编码为 $\boldsymbol{F}$, 通过非局部学习单元(non-local learning unit, NLU)从 $\boldsymbol{F}$ 逐步提取噪声特征图 $\Delta \boldsymbol{F}$; 再 使用另一组共享的 MLP 对噪声降维; 最后, 从噪声 点云中 $\mathcal{P}$ 减去噪声位移 $\mathcal{N}$ 得到去噪后的结果 $\mathcal{Q}$.

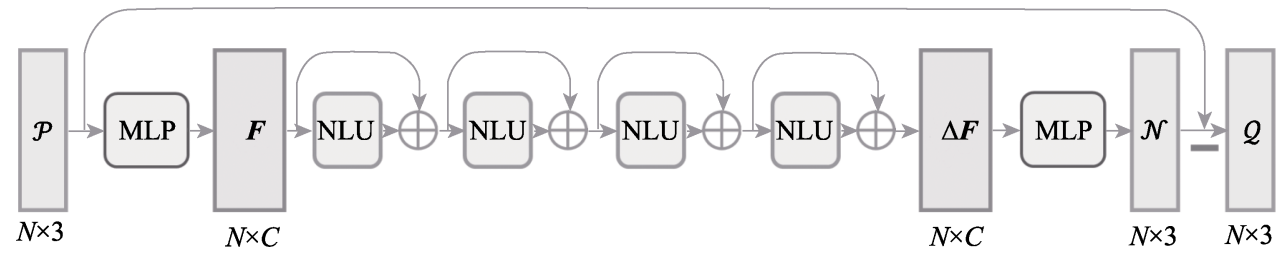

图 9 NLPA 去噪重建网络结构 ${ }^{[19]}$

\section{3 点云修复网络模型中的关键技术}

特征编码、特征扩展及用于神经网络训练的损 失函数等是基于深度学习的点云修复网络模型的 重要组成部分, 其设计在一定程度上决定了模型 的最终性能. 本节将对三维点云分析与处理中常 用的特征编码、特征扩展和损失函数等进行分析, 并总结点云修复中常用的典型网络模块和点云数 据集.

\section{1 特征编码}

特征编码的目的是从输人点集 $P(N \times d)$ 中提取 特征向量. 三维点云的特征编码是点云修复中的关 键技术，也是三维点云分类及语义分割的基础，近 几年涌现出了大量用于点云特征学习的深度神经网
络, 如 PointNet ${ }^{[12]}$, PointNet $++^{[13]}$, SpiderCNN ${ }^{[43]}$, SplatNet $^{[44]}$, PointCNN ${ }^{[45]}$, PointConv ${ }^{[46]}$, PointWeb ${ }^{[47]}$, DGCNN $^{[48]}$ 和 PointSIFT ${ }^{[49]}$ 等.

下面简要介绍三维点云处理技术(包括点云修 复技术)中常用的几种特征编码方法.

\subsection{1一般编码}

在全局特征编码中, MLP 通常用于对输人点 云数据的特征编码, 最大池化操作用于将 $N \times D$ 的 特征矩阵聚合, 最终将其编码成 $1 \times D$ 全局特征向 量, 如图 10 所示. 例如, PointNet ${ }^{[12]}, \mathrm{PCN}^{[10]}$ 和文献 [39]都采用 MLP 和最大池化策略进行特征编码. 在 PointNet ${ }^{[12]}$ 中, 输人数据先通过与一个预测变 换网络(T-Net)学习到的转换矩阵相乘对齐数据, 保证了模型对特定空间转换的不变性; 通过多次 
MLP 对各点云数据进行特征提取后, 再用一个 T-Net 对特征进行对齐; 最后在特征的各个维度上 执行最大池化操作得到最终的点云特征 $\boldsymbol{F} . \mathrm{PCN}^{[10]}$ 中, 基于 stacked PointNet 的编码器包括 2 层: 第 1 层在 ReLU 激活的情况下, 使用一个共享的 MLP 将每个点坐标转换成一个点特征向量 $\boldsymbol{F}$, 然后, 在 所有点的特征矩阵上执行基于点的最大池化, 得 到一个 $K$ 维全局特征 $\boldsymbol{G}$; 第 2 层将 $\boldsymbol{G}$ 和 $\boldsymbol{F}$ 拼接作 为一组共享 MLP 的输人, 执行基于点的最大池化 得到输人点集的特征 $\boldsymbol{V}$.

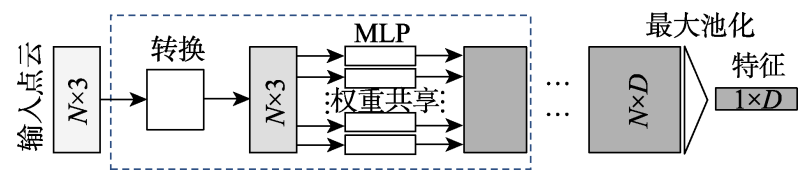

图 $10 \mathrm{MLP}+$ 最大池化进行特征编码

全局特征编码容易丢失局部特征信息. 多层 次特征提取可以有效地融合不同尺度的特征．例 如, PointNet $++^{[13]}$ 构建了点的层次分组, 并沿着层 次逐步抽象出越来越大的局部区域. 该模型首先 采用迭代最远距离采样算法从输人点云中抽取 $M$ 个点; 然后采用半径为 $r$ 的球体、以每个选定的点 为中心捕获点云块，从中学习不同尺度的特征; 利
用 PointNet 将可变点数转换为定长局部区域特征 $N \times C$; 经过多级操作, 最后拼接所有的特征得到特 征编码 $1 \times C^{\prime}$. 文献[22]的特征编码器由大小为 1 的 卷积核构成的一维卷积层、批量归一化层和 ReLU 层组成, 卷积的权值和偏差由所有点共享. 在编码 器的最后一层应用最大池化生成一个潜在的特征 向量. 神经网络浅层特征一般反映局部的小尺度 特征, 反之亦然. PU-Net ${ }^{[20]}$ 随机选择点云物体表面 的 $M$ 个点, 可同时从点云块学习局部与全局的几 何信息. 从每个选定的点生成一个个曲面的小块, 使用泊松圆盘采样算法在每个小块上随机生成 $N$ 个点, 通过 PointNet 获得多级特征, 用插值恢复 $N$ 个点不同层次的特征, 最后将不同尺度的特征串联 形成多尺度特征. PU-GAN ${ }^{[18]}, 3 P U^{[21]}$ 和 PU-GCN ${ }^{[11]}$ 均是使用密集连接模块来集成不同层之间的特征. 例如, $3 \mathrm{PU}^{[21]}$ 在密集块内部和块之间引人密集连 接, 如图 11 所示. 在密集块内部, 每个 MLP 的输 出如固定尺度的特征, 被传递给所有后续的 MLP; 在密集块之间，每个块产生的特征被作为输人提 供给所有后续块; 通过多级密集块和密集连接最终 得到特征编码. Qian 等 ${ }^{[11]}$ 引人了基于 GCN 的特征编 码方法, 用一个 Inception DenseGCN 模块编码来自 DenseGCN ${ }^{[25]}$ 的多尺度点云特征.

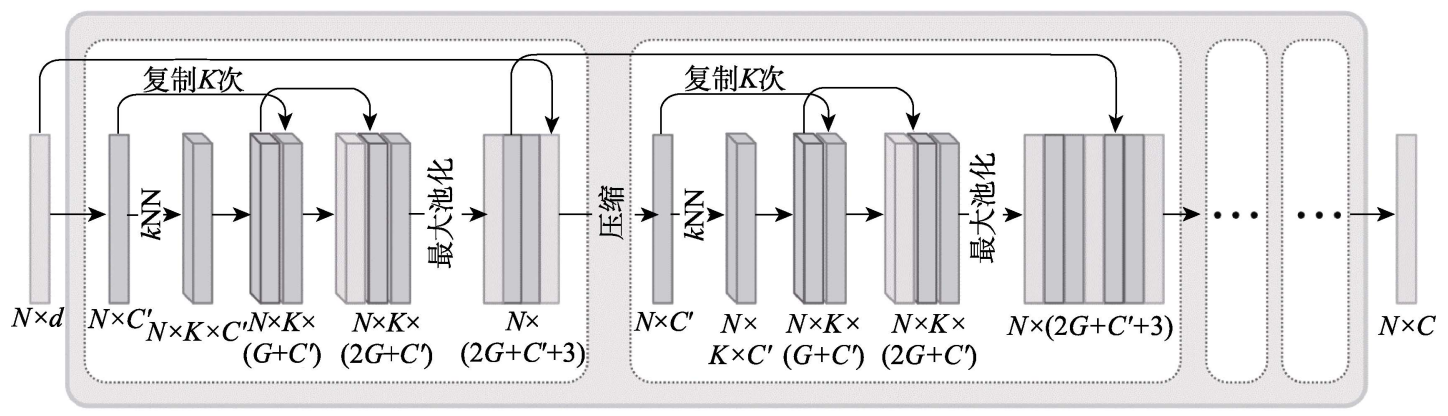

图 11 密集连接特征编码 ${ }^{[21]}$

\section{1 .2 旋转不变性特征编码}

在实际应用中，由于点云模型的方向通常是 先验未知的, 现有的很多点云特征编码无法应用 于旋转形状分析. 此外, 现有的框架需要扩充大量 的数据处理旋转, 会导致不可接受的计算成本. 球 形卷积和提取标量特征是目前常用的 2 类旋转不 变特征提取方法.

球形卷积 ${ }^{[50-51]}$ 实现了从连续网格中提取全局 特征的算法. Cohen 等 ${ }^{[50]}$ 通过快速傅里叶变换求解 球面体素卷积避免过度计算。文献[52]采用球面体 素卷积和点重采样提取每个点的旋转不变特征, 如图 12 所示, 以稀疏点作为输人，通过多个球形体
素卷积层形成一个球形体素网格, 然后把这些体素 最大池化得到一个适合分类的全局特征. Poulenard 等 ${ }^{[53}$ 将基于球面谐波核函数和基于点的旋转不变 性网络融人点 CNN 架构中, 以保证全局形状转换 和局部旋转的不变性.

除了基于球面思想的旋转不变特征编码方式 外, 很多算法通过提取相对标量特征来保证旋转 不变性. Sun 等 ${ }^{[54]}$ 提出了点投影特征算法, 即选择 输人对象的 3 个主轴, 将每个点投射到这些轴上, 以获得一个三维特征以及向量从中心点到这点的 范数. 因为点与所选轴的相对位置关系在旋转时 保持不变, 最终的编码特征与输人点云的方向无 


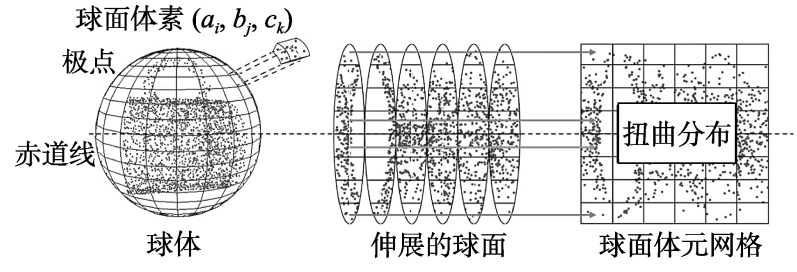

图 12 球形坐标下的扭曲分布 ${ }^{[52]}$

关. Li 等 ${ }^{[55]}$ 将局部和全局信息构成的低阶旋转不 变量表示(距离、角度等)代替常用的三维笛卡儿坐 标作为网络输人, 并用一种基于 MLP 和区域关系 卷积的深层次网络架构将这些表示嵌人特征中.

\section{2 特征扩展}

在三维修复技术中, 提取出点云特征后通常 要进行特征扩展, 即将 $N \times C$ 维的特征扩展成 $r N \times C$ 维, 通过扩展特征空间中的特征数量达到扩展点 的数量的目的. 其中, $C$ 为每个点的特征数目, $N$ 为 输人点的个数, $r$ 为提升的分辨率的倍数(上采样 率). 特征扩展模块的性能会影响最终网络的有效 性. 例如, PU-Net ${ }^{[20]}$ 使用了基于子像素卷积层的特 征扩展操作对提取的多层级聚合特征进行扩展; Qian 等 ${ }^{[11]}$ 提出了基于 GCN 的 Multi-branch GCN, Clone GCN 和 NodeShuffle3 个点云上采样模块扩 展点云特征; $3 \mathrm{PU}^{[21]}$ 采用了点特征复制的方法. 由 于多分支特征扩展容易丢失邻域信息，而点特征 复制易于生成类似于原始点云的点云块, 因此 PU$\mathrm{GAN}^{[18]}$ 设计了如图 13 所示上下扩展单元, 以增强 特征扩展中的特征变化, 从而使生成器能够产生 更多样化的点分布.

\section{3 损失函数设计}

在损失函数计算中，常用的距离函数包括 Chi-

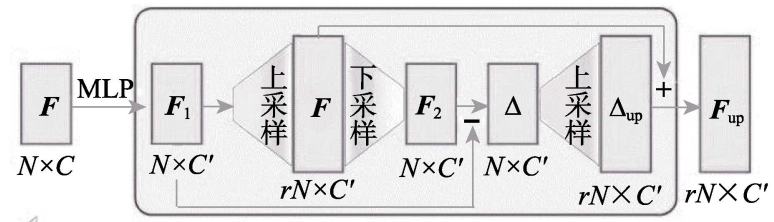

特征上采样操作
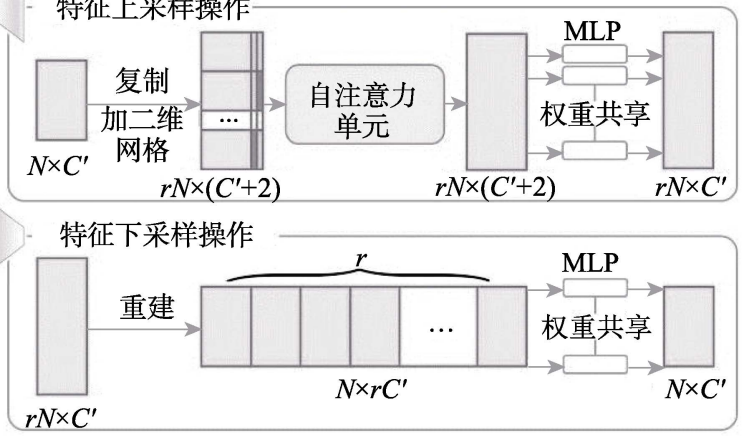

图 13 PU-GAN 特征扩展 ${ }^{[18]}$ squared 模型、 $L_{1}$ 范数、 $L_{2}$ 范数和倒角距离 (chamfer distance, CD), 推土机距离 (earth mover's distance, EMD), 豪斯多夫距离(Hausdorff distance, HD)等. 其中, $\mathrm{CD}^{[56]}$ 和 $\mathrm{EMD}^{[57]}$ 是三维点云修复技术中最常 用的损失函数计算形式 ${ }^{[22]}$.

\subsection{1 重建精度}

重建精度损失函数中常用的 2 种距离函数包

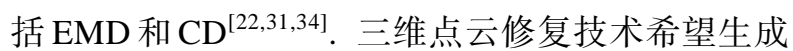
的点能和真值点云中的点位置一一匹配. 利用 EMD 函数建立生成点云 $A$ 到真值点云 $B$ 的双射映射, 使 $A$ 中所有点到 $B$ 中对应点的距离之和最小, 即 $\mathcal{L}_{r 1}=\min _{\phi: A \rightarrow B} \sum_{x_{i} \in A}\left\|x_{i}-\phi\left(x_{i}\right)\right\|_{2}$. 其中, $\phi$ 为 2 个相同大 小点云 $A$ 和 $B$ 之间的双射映射.

同时，三维点云修复技术希望生成的点都位 于目标表面上. 利用 $C D$ 函数对于 $A$ 中的每个点, 求得 $B$ 中离它距离最近的一个点, 使所有点距离 之和最小. 再对 $B$ 中的每个点, 求得 $A$ 中离它距离 最近的一个点, 使点距离之和最小. 二者相加作为 另外一个重建精度损失函数, 即

$$
\mathcal{L}_{r 2}=\frac{1}{|A|} \sum_{x \in A} \min _{y \in B}\|x-y\|_{2}+\frac{1}{|B|} \sum_{y \in B} \min _{x \in A}\|y-x\|_{2} .
$$

为了减少噪声和异常值, 也有文献提出了修 改版的 $\mathrm{CD}$ 损失函数 ${ }^{[11,20-21]}$. 例如, 在 $3 \mathrm{PU} \mathrm{U}^{[21]}$ 中, 将 $\mathrm{CD}$ 损失函数重新定义为

$$
\begin{gathered}
\mathcal{L}_{r 2^{\prime}}=\frac{1}{|A|} \sum_{x \in A} \xi\left(\min _{y \in B}\|x-y\|^{2}\right)+ \\
\frac{1}{|B|} \sum_{y \in B} \xi\left(\min _{x \in A}\|x-y\|^{2}\right) .
\end{gathered}
$$

\subsection{2 排斥损失}

排斥损失用来激励生成的点在物体表面相互远 离, 使点更均匀地分布在物体表面上. 给定一组输 出点 $x_{i}, i=1,2, \cdots N$, 排斥损失函数 $\mathcal{L}_{c}$ 定义 ${ }^{[19]}$ 为 $\mathcal{L}_{c}=\frac{1}{N \cdot k} \sum_{i=0}^{N} \sum_{i^{\prime} \in K(i)} \eta\left\|x_{i}^{\prime}-x_{i}\right\|$. 其中, $K(i)$ 为点 $x_{i} \in A$ 的一组 $k$ 邻近点的索引; $\eta(r)=\max \left(0, h-r^{2}\right)$ 为惩罚 权重; $h$ 由经验值给定或某种规则计算. 通常只有 在 $x_{i}$ 太接近某些邻点时才进行惩罚, 所以在实际 计算中只考虑 $x_{i}$ 周围几个最近的邻点. 此外, 当点 对点距离大于 $h$ 时, 可考虑消除斥力效应 ${ }^{[41]}$.

\subsection{3 均匀约束}

PU-GAN ${ }^{[18]}$ 设计了全局和局部 2 个均匀约束 损失函数. 在生成点云集合 $A$ 中, 通过最远距离采 样等采集 $M$ 个种子点, 在每个以 $M$ 为中心 $r$ 为 
半径的球体 $p$ 中, 归一化每个点的大小, 求得球体 $p$ 中期望包含的点的个数 $N$ 与球体 $p$ 中实际包含 的点的个数 $N^{\prime}$ 的差值，作为全局均匀约束损失函 数 $\mathcal{L}_{e 1}$. 同时, 求得 $p$ 中各点与其相邻点的平均距 离 $d$, 将球体内每个点与它最邻近点的距离 $d^{\prime}$ 与 $d$ 的距离差之和作为局部均匀约束损失函数 $\mathcal{L}_{e 2}$, 将 $\mathcal{L}_{e 1}$ 和 $\mathcal{L}_{e 2}$ 之和作为总的均匀约束损失函数 $\mathcal{L}_{e}$.

在实际应用中，通常将以上全部或几种损失 函数的加权和作为最终的损失函数, 即

$$
\mathcal{L}=\alpha \mathcal{L}_{r}+\beta \mathcal{L}_{c}+\gamma \mathcal{L}_{e} .
$$

其中, $\alpha, \beta, \gamma$ 为权重.

此外, 还有文献使用了均方误差(mean square error, MSE)、余弦相似度的绝对值等作为损失函数 ${ }^{[29]}$.

\section{4 典型网络模块}

在基于深度学习的点云修复技术中, 很多神 经网络模型常常作为子模块嵌人点云修复网络中, 辅助网络进行特征提取或特征增强. 常用模块包 括 PointNet, PointNet++, GCN, 折叠网络和注意力 网络等.

PointNet 模型 ${ }^{[12]}$ 首先将深度学习技术引人点 云处理中, 以一组无序点作为输人自动学习用于 对象分类和分割的特征, 并验证了它能够用于点 云数据的多种认知任务, 如分类、语义分割和目标 识别等. 由此, PointNet 或其部分结构或其扩展模 块常常应用到三维点云修复模型中作为特征提取 或特征提取的一部分 ${ }^{[10,20,34]}$. PointNet++模型 ${ }^{[13]}$ 能 够在不同尺度提取局部特征，通过多层网络结构 得到深层特征，它的多层次特征提取技术也常常 被应用到点云修复模型中 ${ }^{[20,35-36,41]}$

GCN 是一种神经网络架构, 它可以利用图的 结构, 以卷积的方式从邻域聚集节点信息, 如图 14 所示. GCN 能够对不规则离散数据进行有效的 卷积处理, 逐渐成为三维无序离散点云处理中的 研究热点 ${ }^{[58-59]}$, 它也常常被用于点云密集重建和 去噪重建的特征编码中 ${ }^{[11,37]}$.

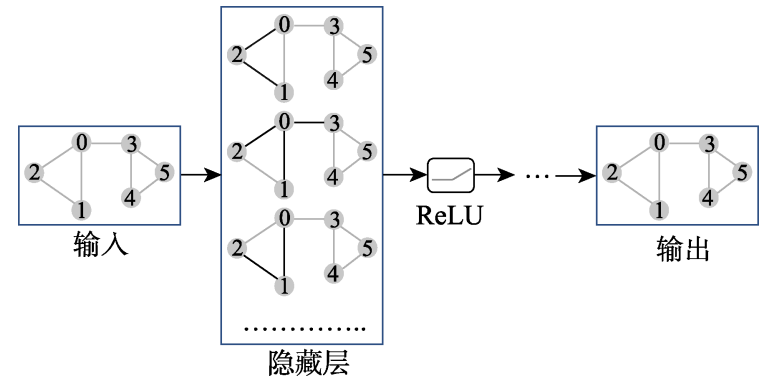

图 14 GCN
任何三维物体表面都可以通过切割、挤压和拉 伸等特定操作转化为二维平面 ${ }^{[29]}$. 基于这个思想, 折叠网络实现了三维到二维转化的逆过程, 即引 人隐含的二维网格约束, 通过一个 $N(N=3)$ 层感 知器来重建点云结构. 如图 15 所示, 将输人为 $1 \times C$ 的特征向量复制 $m$ 次得到一个 $m \times C$ 的特征 矩阵, 此矩阵与 $t \times t$ 的方形网格中的 $m(t \times t=m)$ 个 点坐标向量 $m \times 2$ 拼接成 $m \times(C+2)$ 的特征矩阵, 最后通过 3 层的感知器得到 $m \times 3$ 的点云. 折叠网 络由于在还原物体真实的局部几何形状方面的独 有优势, 因此常常被应用在三维点云修复模型的 解码器中 ${ }^{[10,31,35-36]}$

折叠解码器

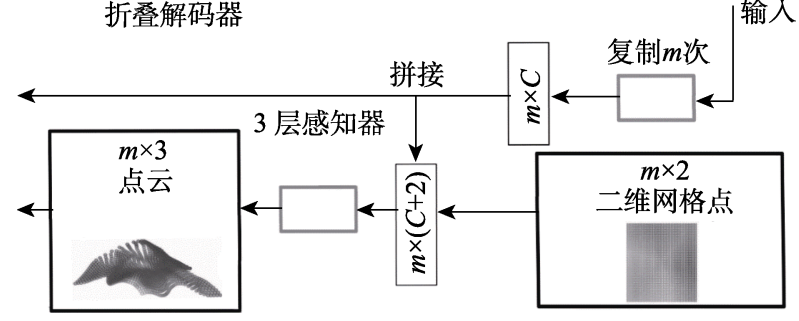

图 15 折叠网络 ${ }^{[29]}$

基于深度学习的点云修复模型中常用的注意 力网络主要有生成权值注意力网络和非局部注意 力网络 2 类. 如图 16 所示, 生成权值注意力网络模 块 ${ }^{[31,35]}$ 为每个点特征计算权重或分数, 旨在对异 常点或噪声等不重要的点分配较低的权值, 对能 更有效地表征曲面形状的重要点分配较高的权值. 典型的生成权值注意力网络将输人特征通过一组 MLP, 再结合 Softplus 等激活函数最终生成一个和 输人特征相同大小的权重向量(分值表). 通过选择 权值最大的前 $n \%$ 的特征，或者将权值向量与输人 特征相乘, 达到突出或强化更有用特征并降低或 减少无用特征的目的.

卷积核通常是局部的，在基于 CNN 技术的计

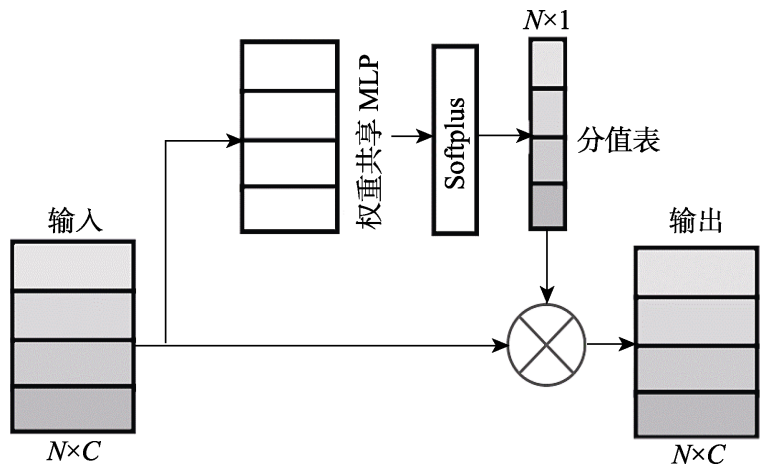

图 16 生成权值注意力网络 
算机视觉处理任务中常常由于语义信息不足而影 响最终的处理结果. 为了增加卷积操作感受野, 受 计算机视觉中经典的非局部均值算法的启发, 如 图 17 所示, 非局部神经网络 ${ }^{[60]}$ 通过计算三维点云 任意 2 个位置之间的交互捕捉非局部感受野信息, 以增强特征表示. 此类模块被广泛应用于三维点 云处理 ${ }^{[18-19,36]}$.

以图 18 所示 PU-GAN ${ }^{[18]}$ 中的非局部注意力网 络模块为例, 输人特征 $\boldsymbol{F}$ 首先分别通过 3 组 MLP 转换为 $\boldsymbol{G}, \boldsymbol{H}$ 和 $\boldsymbol{K}$, 然后由 $\boldsymbol{G}$ 和 $\boldsymbol{H}$ 生成注意力权值 $\boldsymbol{W}\left(\boldsymbol{W}=f_{\text {Softmax }}\left(\boldsymbol{G}^{\mathrm{T}} \boldsymbol{H}\right)\right)$, 计算加权特征 $\boldsymbol{W}^{\mathrm{T}} \boldsymbol{K}$; 最后输 人特征和加权特征相加得到输出特征.

\section{5 常用数据集}

三维点云修复中常用的数据集包括 Visionair repository, ShapeNet, ScanNet 和 KITTI 等. 表 1 所 示为这些数据集在三维点云修复中用到的点云性 质、特点及在相关点云修复文献中的使用情况. 下 面简要介绍常用的数据集.

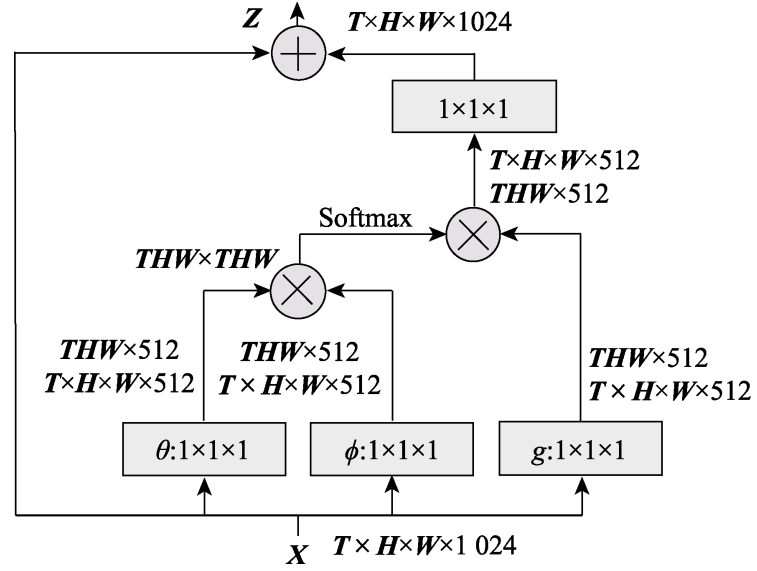

图 17 非局部神经网络结构 ${ }^{[60]}$

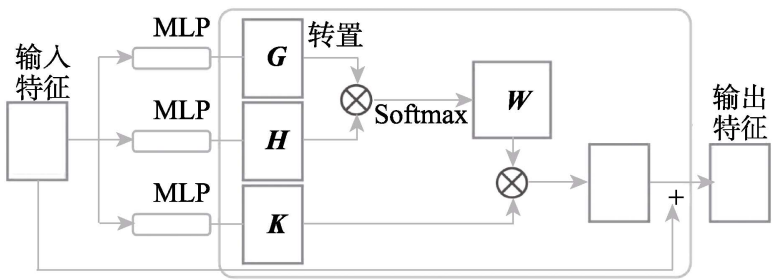

图 18 点云修复中的非局部网络 ${ }^{[18]}$

表 1 点云修复中常用的数据集

\begin{tabular}{|c|c|c|c|c|}
\hline \multirow{2}{*}{ 数据集 } & \multirow{2}{*}{ 性质 } & \multicolumn{3}{|c|}{ 点云修复中的相关文献 } \\
\hline & & 密集重建 & 补全重建 & 去噪重建 \\
\hline Visionair repository & 合成 高质量, 多分辨率 & PU-GAN ${ }^{[18]}$, PU-Net $^{[20]}$ & & $\mathrm{NLPA}^{[19]}$ \\
\hline ShapeNet ${ }^{[61]}$ & 合成 规整, 带标注, 网格质量较低 & Zhang 等 ${ }^{[22]}$, PU-Net $^{[20]}$ & $\begin{array}{l}\text { Zhang 等 }{ }^{[31]} \text {, Huang 等 }{ }^{[30]} \text {, } \\
\mathrm{PCN}^{[10]} \text {, Chen 等 } \\
\text { Wang } \\
\text { 等 }\end{array}$ & $\begin{array}{l}\mathrm{NLPA}^{[19]}, \text { Pistilli }^{[37]} \text {, } \\
\text { Duan 等 }^{[39]}, \mathrm{Yu} \text { 等 }^{[41]}\end{array}$ \\
\hline ScanNet $^{[62]}$ & 真实 带标注, 不均匀, 有噪声和空洞 & & Chen 等 ${ }^{[28]}$ & \\
\hline $\mathrm{KITTI}^{[63]}$ & 真实 带标注, 稀疏, 不完整 & PU-GCN ${ }^{[11]}$, PU-GAN ${ }^{[18]}$ & $\begin{array}{l}\mathrm{PCN}^{[10]} \text {, Chen 等 } \\
\text { Zhang } \text { 等 }^{[35]}\end{array}$ & \\
\hline ModelNet $^{[64]}$ & 合成 规整, 结构简单 & $\begin{array}{l}\text { PU-Net }{ }^{[20]}, 3 P U^{[21]} \\
\text { PU-GAN }\end{array}$ & & $\begin{array}{l}\text { Pistilli 等 }{ }^{[37]} \text {, Duan 等 }{ }^{[39]} \text {, } \\
\text { Luo 等 }\end{array}$ \\
\hline Sketchfab & 合成 规整, 高分辨率 & $3 \mathrm{PU}^{[21]}$ & & \\
\hline Paris-rue-Madame ${ }^{[65]}$ & 真实 带标注, 不均匀, 有噪声, 不完整 & & & $\mathrm{NLPA}^{[19]}$, Luo 等[42] \\
\hline SHREC15 ${ }^{[66]}$ & 合成 规整, 需采样点集 & PU-Net ${ }^{[20]}$ & & \\
\hline Matterport3D $\mathrm{D}^{[67]}$ & 真实 带标注, 不均匀, 有噪声和空洞 & & Chen 等[28] & \\
\hline
\end{tabular}

Visionair repository 数据集 ${ }^{(1)}$ 中的点云相对规 整，包含了从光滑的非刚性物体到陡峭的刚性物 体等多种点云模型. 使用者可以根据需要自行选 择三维模型的分辨率. 该数据集常用于密集重建 和去噪重建神经网络模型的训练和测试 ${ }^{[18-20]}$.

ShapeNet ${ }^{[61]}$ 是一个大型的、信息丰富的三维模 型库，它包含跨越多种语义类别的模型，常用的子 集包括 ShapeNetCore，ShapeNetSem 等. 其中，
ShapeNetCore 具有单个干净的三维模型和手动验 证的类别和对齐注释，它涵盖了 55 个常见对象类 别, 约 51300 个独特的三维模型. ShapeNetSem 是 一个更小的、注释更密集的子集，由分布在更广的 270 个类别中的 12000 个模型组成. 此数据集常用 于点云修复深度网络模型的训练和测试.

ScanNet ${ }^{[62]}$ 是一个 RGB-D 数据集, 包含了在 707 个不同空间中捕捉的 1513 个场景的 2.5 万个 
视图, 以及对应的三维摄像机姿态、表面重建以及 语义分割标注.

$\mathrm{KITTI}^{[63]}$ 包含使用了多种传感器模式采集的 市区、乡村和高速公路等场景的真实点云数据, 记 录了 $10 \sim 100 \mathrm{~Hz}$ 下 $6 \mathrm{~h}$ 的交通场景. 视图包括各种 程度的遮挡与截断，数据集分为“道路”“城市”“住 宅”“校园”“人”. 对于每个序列，它提供了原始数 据以及三维包围框形式的对象注释和校准文件, 常用于密集重建及形状补全 ${ }^{[10,18]}$.

ModelNet 数据集 ${ }^{[64]}$ 由普林斯顿视觉与机器人 实验室于 2015 年发布, 包含了 662 种目标分类, 127915 个 CAD 模型, 以及 10 类带方向标记的数 据. 此数据集常用于三维形状分类及检索，在点云 修复中也常用于点云密集重建和去噪重建深度网 络模型的测试 ${ }^{[20,39]}$.

Sketchfab ${ }^{\mathbb{1}}$ 是一个发布、分享、发现、买卖三 维、虚拟现实和增强现实相关内容的平台, 用户可 以选择根据知识共享协议下载三维模型文件. 由 于其中的点云模型质量较高, 常常用于点云修复 模型的训练和测试 ${ }^{[18-20]}$

Paris-rue-Madame ${ }^{[65]}$ 是来自 rue Madame 的公 共三维标记数据集, 它包含了使用移动激光扫描 系统扫描得到的 $160 \mathrm{~m}$ 长的街道段物体点云, 带有 点三维坐标、反射率、对象标签和对象类. 其类别 包括建筑物、地面、汽车、摩托车、行人和交通标 志等, 可用于基准城市检测、分割和分类等，在点 云修复中主要用作模型测试 ${ }^{[19,42]}$.

SHREC 是 Eurographics 举办的每年一届的三 维模型检索大赛. 其中, SHREC15 ${ }^{[66]}$ 数据集包含 了 50 个类别的 1200 个三维三角网格模型. 同一类 别的 24 个模型都是由一个原始的三维网格通过实 现各种姿态转换而生成. 例如, PU-Net 从每类中随 机选择一个模型对点云修复网络模型进行测试 ${ }^{[20]}$.

三维室内场景数据集 Matterport3D ${ }^{[67]}$ 包含 90 个建筑物的 194400 幅 RGB-D 图像、10800 幅全景 图像和 24727520 个纹理三角面，并给出了对应的 彩色图、深度图、全景天空盒、纹理网格、区域布 局和类别以及语义分割. 由于此数据集中点云不 均匀, 且含有噪声和空洞, 因此 Chen 等 ${ }^{[28]}$ 在此数 据集上进行了补全重建.

以上数据集中的合成点云模型往往质量较高, 在点云修复中通过这些数据模型提取低分辨率的 点、或生成部分点云、或加人高斯噪声等生成待修
复点云, 与原始点云 (真值)一起作为点云修复网络 模型的训练集和测试集. 而真实点云数据集由于 存在稀疏、残缺或噪声等问题, 并且缺乏真值, 因 此常常用来对已训练好的模型进行测试.

\section{4 实验结果与评估对比}

本节介绍几种常用点云修复质量评估方法, 并给出几种经典点云修复网络模型的评估对比.

\section{1 常用度量评估方法}

点云修复质量常用的评价函数包括均值 ${ }^{[41]}$ 、均 方根 ${ }^{[41]}$ 和 $\mathrm{MSE}^{[29]}, \mathrm{CD}^{[56]}, \mathrm{EMD}^{[57]}$, NUC(normalized uniformity coefficients) ${ }^{[20]}$, P2F(point-to-surface) ${ }^{[11,18]}$, FPD(Fréchet point cloud distance) ${ }^{[34]}$, RMSD(root mean square distance-to-surface) ${ }^{[40]}$, C2C(cloud-tocloud), HD 等. 其中, 集合 $A$ 到集合 $B$ 的 $\mathrm{HD}$ 是一 个极大极小函数, 定义为

$$
\operatorname{HD}(A, B)=\max _{x \in A}\left\{\min _{y \in B}\{d(x, y)\}\right\} .
$$

FPD 计算点集特征空间中真实高斯测度和伪 高斯测度之间的 2-Wasserstein 距离，即

$$
\operatorname{FPD}(A, B)=\left\|\boldsymbol{m}_{A}-\boldsymbol{m}_{B}\right\|_{2}^{2}+\operatorname{tr}\left(\boldsymbol{\Sigma}_{A}+\boldsymbol{\Sigma}_{B}-2\left(\boldsymbol{\Sigma}_{A} \boldsymbol{\Sigma}_{B}\right)^{\frac{1}{2}}\right) .
$$

其中, $\boldsymbol{m}$ 和 $\boldsymbol{\Sigma}$ 分别代表均值和协方差矩阵; $\operatorname{tr}(\cdot)$ 表示矩阵对角元素之和.

MSE, RMSD 和 C2C 分别定义为

$$
\begin{aligned}
\operatorname{MSE}(A, B) & =\frac{1}{N} \sum_{x \in A, y \in B}\|x-y\|_{2}^{2}, \\
\operatorname{RMSD}(A, B) & =\sqrt{\frac{1}{N} \sum_{x \in A} \min _{y \in B}\|x-y\|_{2}^{2}},
\end{aligned}
$$

$$
\begin{aligned}
& \mathrm{C} 2 \mathrm{C}(A, B)= \\
& \frac{1}{2}\left[\frac{1}{N_{A}} \sum_{x \in A} \sqrt{\min _{y \in B}\|x-y\|_{2}^{2}}+\frac{1}{N_{B}} \sum_{y \in B} \sqrt{\min _{x \in A}\|y-x\|_{2}^{2}}\right] .
\end{aligned}
$$

\section{2 结果评估}

本节给出了部分密集重建、补全重建和去噪重 建模型的量化结果.

(1) 密集重建. 数据集来自 PU-GAN ${ }^{[18]}$ 中的 147 个三维模型, 包括从简单模型(如 20 面体)到复 杂精细模型(如雕像), 随机选取 120 个模型进行训 练, 其余模型进行测试. 结果如表 $2^{[11]}$ 所示. 与 PU-Net ${ }^{[20]}$ 相比, 3PU ${ }^{[21]}$, PU-GAN ${ }^{[18]}$ 和 PU-GCN ${ }^{[11]}$ 在 CD 和 $\mathrm{HD}$ 度量上取得了较好的效果.

(1) https://sketchfab.com 
表 2 密集重建模型比较 ${ }^{[11]}$

\begin{tabular}{|c|c|c|c|c|c|c|}
\hline \multirow{2}{*}{ 网络模型 } & \multicolumn{3}{|c|}{$10^{3} \times$ 输人稀疏点云数 256} & \multicolumn{3}{|c|}{$10^{3} \times$ 输人稠密点云数 1024} \\
\hline & CD & HD & $\mathrm{P} 2 \mathrm{~F}$ & $\mathrm{CD}^{3}$ & HD & $\mathrm{P} 2 \mathrm{~F}$ \\
\hline PU-Net ${ }^{[20]}$ & 2.461 & 14.370 & 13.099 & 0.720 & 8.940 & 6.840 \\
\hline $3 P U^{[21]}$ & 2.177 & 12.672 & 10.328 & 0.490 & 6.110 & 3.960 \\
\hline PU-GAN ${ }^{[18]}$ & 2.072 & 16.592 & 8.055 & 0.280 & 4.640 & 2.330 \\
\hline PU-GCN ${ }^{[11]}$ & 1.973 & 11.786 & 9.591 & 0.254 & 1.821 & 2.935 \\
\hline
\end{tabular}

(2) 补全重建. 数据集采用 ShapeNet 数据集, 训练集包含 28974 个不同的模型, 对飞机、樞柜、 汽车、椅子、灯、沙发、桌子和船 8 类点云模型的
测试结果如表 $3^{[31]}$ 所示, 其度量为 $C D \times 10^{4}$. 根据 量化结果可以看出, MSPCN w/ MVCS 优于其他形 状补全重建模型.

表 3 补全重建模型定量比较 $\left(C D \times 10^{4}\right)^{[31]}$

\begin{tabular}{|c|c|c|c|c|c|c|c|c|c|}
\hline \multirow{2}{*}{ 网络模型 } & \multicolumn{8}{|c|}{ 点云模型 } & \multirow{2}{*}{ 平均 } \\
\hline & 飞机 & 檘柜 & 汽车 & 椅子 & 灯 & 沙发 & 桌子 & 船 & \\
\hline $\mathrm{FC}^{[12]}$ & 5.70 & 11.02 & 8.78 & 10.97 & 11.13 & 11.76 & 9.32 & 9.72 & 9.80 \\
\hline Folding ${ }^{[30]}$ & 5.97 & 10.91 & 9.32 & 11.20 & 12.02 & 11.53 & 9.37 & 9.82 & 10.02 \\
\hline $\mathrm{PCN}^{[10]}$ & 5.51 & 10.62 & 8.68 & 11.00 & 11.34 & 11.68 & 8.59 & 9.67 & 9.64 \\
\hline TopNet ${ }^{[32]}$ & 5.85 & 10.78 & 8.84 & 10.80 & 11.15 & 11.41 & 8.79 & 9.29 & 9.62 \\
\hline MSPCN w/ MVCS ${ }^{[31]}$ & 5.59 & 10.39 & 8.79 & 10.78 & 10.72 & 11.19 & 8.42 & 9.28 & 9.39 \\
\hline
\end{tabular}

（3）去噪重建. 测试了 ShapeNet 中添加了白 噪声的 10 个点云模型(标准差 $\sigma=0.02$ ), 结果如表
$4^{[37]}$ 所示, 其度量为 C2C $\times 10^{3}$. GraphPointNet 结果优 于 GLR 和基于深度学习的 PointCleanNet 等模型.

表 4 去噪重建模型定量比较 $\left(\mathrm{C} 2 \mathrm{C} \times 10^{3}\right)^{[37]}$

\begin{tabular}{lccccccccrrr}
\hline \multirow{2}{*}{ 网络模型 } & \multicolumn{10}{c}{ 点云模型 } \\
\cline { 2 - 11 } & 飞机 & 长登 & 汽车 & 椅子 & 灯 & 枕头 & 步枪 & 沙发 & 扬声器 & 桌子 \\
\hline GLR $^{[68]}$ & 7.470 & 7.329 & 7.383 & 7.748 & 7.990 & 7.637 & 7.268 & 8.005 & 7.874 & 7.264 \\
PointCleanNet $^{[40]}$ & 6.253 & 6.481 & 8.754 & 7.722 & 6.393 & 7.274 & 5.563 & 8.960 & 10.561 & 8.348 \\
GraphPointNet $^{[37]}$ & 5.491 & 5.422 & 6.774 & 6.691 & 5.353 & 5.407 & 5.855 & 7.039 & 7.011 & 6.865 \\
\hline
\end{tabular}

\section{5 讨论与展望}

点云修复技术旨在修复三维传感器等采集到 的稀疏的、不完整的、带噪声的和不均匀的数据, 重建高质量的点云. 目前出现的很多技术可以根 据修复任务构建有效的深度学习网络进行点云重 建，如密集重建、补全重建和去噪重建. 本文全面 总结了近年来相关三维点云修复技术的研究进展. 需要注意的是, 3 种修复技术之间没有明显的边界, 有的可能涵盖了其中修复性重建技术中的 2 3 种. 例如, 在点云补全修复中, 不完整点云常常伴随着 稀疏或低分辨率问题 ${ }^{[10]}$, 所以修补点云形状的同 时也要完成密集重建. 对于这种多任务处理, 点云 补全修复模型在第 1 阶段首先对输人 $N \times 3$ 的点云进 行特征提取, 主要有使用 MLP 和最大池化策略 ${ }^{[0-31]}$, 并伴随特征的复制或拼接; 以及使用 PointNet++
进行多层特征编码 ${ }^{[35-36]}$. 第 2 阶段的解码操作首先 通过 MLP 等模块完成由第 1 阶段得到的特征向量 向点云三维坐标点的转换, 并通过折叠网络等模 块进行细化和上采样等重建操作, 最后输出大小 为 $r N \times 3$ 的完整、均匀的高分辨率点云. 通过补全 重建, 输人点云缺失部分得到了补全, 稀疏部分的 密度得到了提升, 并通过损失函数确保没有或尽 量减少噪声点的产生.

虽然基于深度学习的点云修复技术取得了很 大的成功，但是仍然面临很多的问题和挑战. 第一, 如何保持输人数据旋转不变性. 其中一个普遍的 问题是输人点云数据的旋转不变性得不到有效的 保证，即同一点云和它任意旋转后的点云会提取到 不同的形状特征，由此会导致对任意方向的推广效 果很差. 虽然现在出现了很多三维点云旋转不变特 征的提取算法(如第 3.1.2 节所述), 但仍然有待进一 
步融人基于深度学习的三维点云修复技术中.

第二, 如何修复或保持点云模型的细节信息, 如边缘、角点等尖锐特征和空洞等. 与点云真值相 比, 大多数技术修复点云的局部细节能力欠缺, 如 图 19 所示. 在现有的基于深度学习的点云修复技 术中, 重点考虑细节特征保持的技术还很少. 其 中, EC-Net ${ }^{[41]}$ 通过基于深度学习的边缘感知算法 增强点云边缘信息, 保证了点云边缘细节特征的
修复. 此外, 精确修复三维点云的内部空洞也是目 前三维点云修复技术的挑战, 如图 20 所示. 因为 在修复技术中，点云往往被设计成一个局部均匀 分布的点云, 仅靠损失函数很难完成空洞的准确 修复, 需要研究更好的基于输人密度的上采样及 细节保持算法, 以提高重建精度. 总之, 如何充分 地利用已知信息进行细节信息的保持或修复，仍 是点云修复中需要进一步研究的重点.
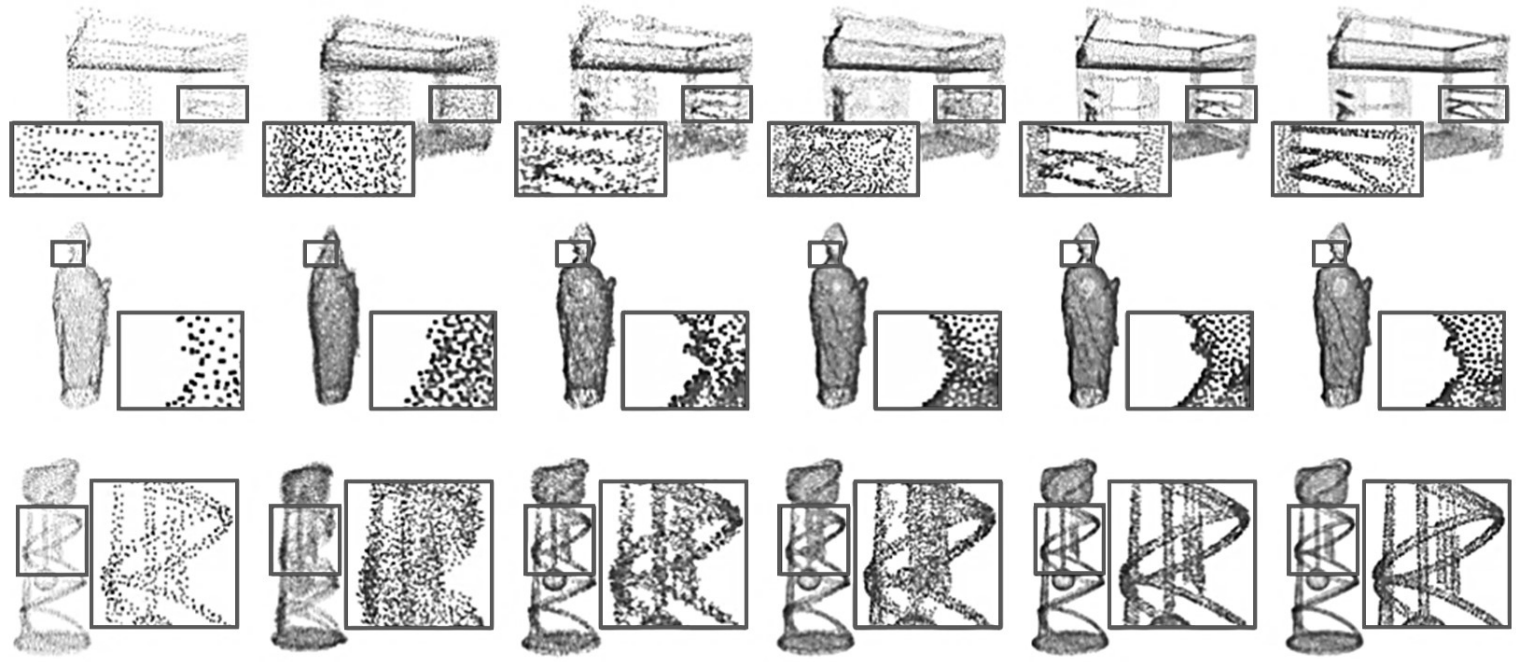

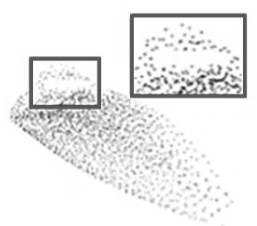

a. 输人点云

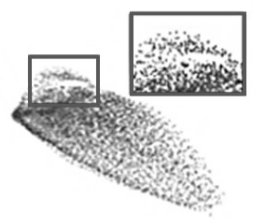

b. PU-Net ${ }^{[20]}$

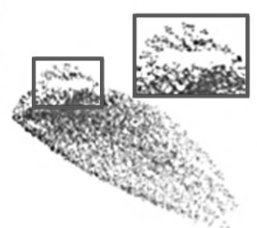

c. $3 P U^{[21]}$

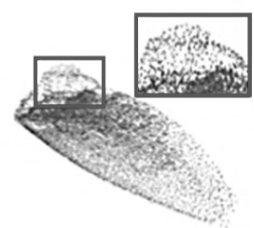

d. PU-GAN ${ }^{[18]}$

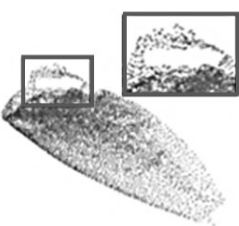

e. PU-GCN ${ }^{[11]}$

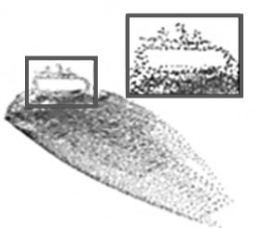

f. 点云真值

图 19 不同网络模型对部分三维点云修复结果 ${ }^{[11]}$

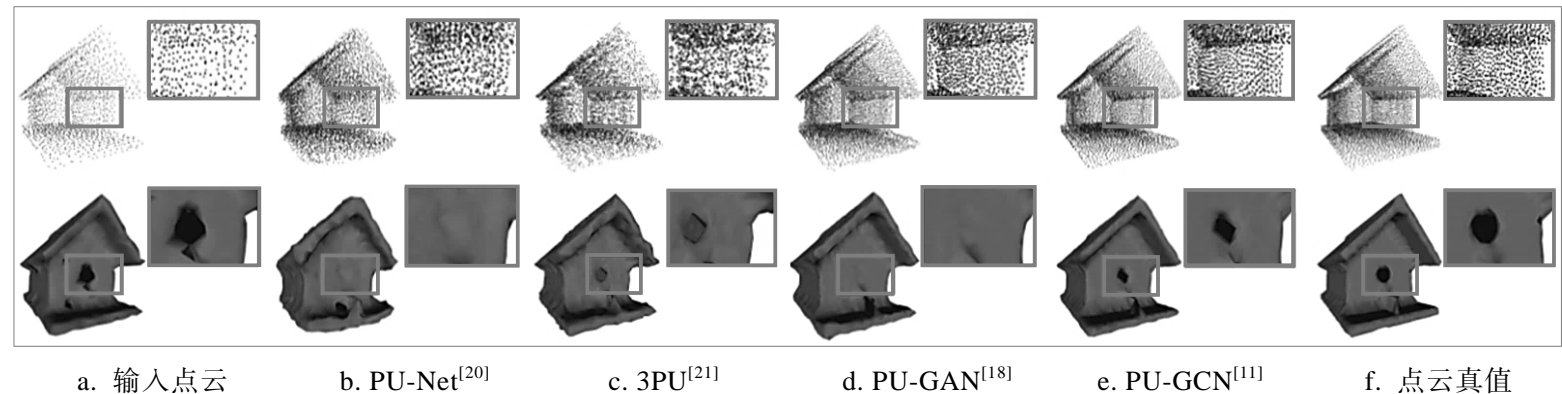

图 20 不同网络模型对鸟巢三维点云修复结果 ${ }^{[11]}$

第三，如何在进行点云修复的同时保持其拓 扑关系. 这里的拓扑是指点云所表示的物体形状 本身的结构，或构成物体形状的基本图元在空间 上的位置关联. 提取拓扑特征，重建点云物体的拓 扑关系, 是点云修复的重要任务. 例如, Tagliasacchi 等 ${ }^{[69]}$ 提出了一种从不完整或残缺的点云中提取
完整的曲线骨架的方法捕获待修复点云的拓扑信 息, 并开发了骨架驱动的点云补全方案完成残缺 点云的形状补全. 在基于深度学习的点云处理中, Chen 等 ${ }^{[70]}$ 提出了基于图拓扑推理和过滤的深度无 监督学习网络模型重建紧凑点云, 将拓扑关系的 重建融人基于深度学习的点云修复技术中, 这是 
提高点云修复准确率的重要途径之一.

第四，如何与传统几何算法相结合，促进三维 深度学习效果. 在三维点云处理中, 有效地运用几 何信息能突出数据中的特定特征，增强数据表示. 如在传统的点云处理技术中, Alexa 等 ${ }^{[71]}$ 利用切空 间的沃罗诺伊图进行点云上采样; Sarkar 等 ${ }^{[72]}$ 使用 基于网格四边形定位的局部块形状编码算法进行 点云物体表面区域修复; $\mathrm{Lu}$ 等 $^{[73]}$ 提出一种基于高 斯混合模型的局部最优投影算法，结合输人点云 的法线信息进行基于特征保持的点云去噪. 但是, 传统点云修复技术往往过度依赖先验和假设，无 法很好地泛化处理不同的输人; 而基于深度学习 的点云处理技术具有很强的自动学习能力和泛化 能力，但缺乏物体表面局部特征信息的有效表达. 因此, 基于深度神经网络的三维点云处理技术开 始与传统几何算法相结合. 例如, Qian 等 ${ }^{[74]}$ 提出的 PUGeo-Net 将离散微分几何融人深度学习中, 以 更好地表达点云局部几何特征. 将传统算法与深 度学习技术相结合, 通过优势互补增强物理理解, 提高点云修复效果, 既是挑战, 也是三维点云修复 技术的发展趋势。

第五，如何利用颜色、法向等信息辅助三维点 云修复. 多模态数据融合能有效地丰富特征表示, 提高基于深度学习的数据处理效果 ${ }^{[75]}$. 近年来, 颜色、深度和边缘等多模态特征在 RGB-D 图像相 关的重建技术中已经得到了广泛应用。例如， Zhang 等 ${ }^{[76]}$ 以 RGB 图像为输人, 从二维图像颜色 信息中预测表面法线和边缘信息，这些信息最终 与 RGB-D 相机提供的原始深度观测数据相结合, 通过深度神经网络完成深度图的修复. Qiu 等 ${ }^{[77]}$ 将 RGB 图像和其对应的稀疏深度图像作为一个深度 神经网络模型的输人，综合运用颜色信息和生成 的法向量信息, 以及学习到的置信模板, 重建完整 密集的深度图. 此类重建技术为广义上的三维点 云重建提供了新的思路，即将多模态特征融合技 术拓展到更普遍的三维点云深度学习中, 辅助点 云坐标信息进行点云修复, 也必将是能有效地提 高点云修复效果的一个新的研究热点.

\section{6 结 语}

高质量的三维点云修复对基于三维点云的后 续处理，如准确的形状分类、物体检测和语义场景 分割等有重要影响. 随着人工智能技术的不断发 展, 基于深度学习的新型神经网络模型不断涌现,
三维点云修复技术也必将迎来更进一步的突破和 发展.

\section{参考文献(References):}

[1] Pfister H, Gross M. Point-based computer graphics[J]. IEEE Computer Graphics and Applications, 2004, 24(4): 22-23

[2] Kobbelt L, Botsch M. A survey of point-based techniques in computer graphics[J]. Computers \& Graphics, 2004, 28(6): 801-814

[3] Rusu R B, Cousins S. 3D is here: point cloud library (PCL)[C] //Proceedings of the IEEE International Conference on Robotics and Automation. Los Alamitos: IEEE Computer Society Press, 2011: 1-4

[4] Aldoma A, Marton Z C, Tombari F, et al. Tutorial: point cloud library: three-dimensional object recognition and 6 DOF pose estimation[J]. IEEE Robotics \& Automation Magazine, 2012, 19(3): 80-91

[5] Saval-Calvo M, Orts-Escolano S, Azorin-Lopez J, et al. A comparative study of downsampling techniques for non-rigid point set registration using color[C] //Proceedings of the International Work-conference on the Interplay Between Natural and Artificial Computation. Heidelberg: Springer Press, 2015: 281-290

[6] Xu Z H, Wu L X, Shen Y L, et al. Tridimensional reconstruction applied to cultural heritage with the use of camera-equipped UAV and terrestrial laser scanner[J]. Remote Sensing, 2014, 6(11): 10413-10434

[7] Chen X Z, Ma H M, Wan J, et al. Multi-view 3D object detection network for autonomous driving[C] //Proceedings of the IEEE Conference on Computer Vision and Pattern Recognition. Los Alamitos: IEEE Computer Society Press, 2017: 1907-1915

[8] Varley J, DeChant C, Richardson A, et al. Shape completion enabled robotic grasping[C] //Proceedings of the IEEE International Conference on Intelligent Robots and Systems. Los Alamitos: IEEE Computer Society Press, 2017: 2442-2447

[9] Berger M, Tagliasacchi A, Seversky L, et al. State of the art in surface reconstruction from point clouds[C] //Proceedings of Eurographics 2014 - State of the Art Reports. Aire-la-Ville: Eurographics Association Press, 2014: 161-185

[10] Yuan W T, Khot T, Held D, et al. PCN: point completion network[C] //Proceedings of the International Conference on 3D Vision. Los Alamitos: IEEE Computer Society Press, 2018: 728-737

[11] Qian G C, Abualshour A, Li G H, et al. PU-GCN: point cloud upsampling using graph convolutional networks[OL]. [2020-11-28]. https://arxiv.org/abs/1912.03264

[12] Qi C R, Su H, Mo K C, et al. PointNet: deep learning on point sets for 3D classification and segmentation[C] //Proceedings of the IEEE Conference on Computer Vision and Pattern Recognition. Los Alamitos: IEEE Computer Society Press, 2017: $652-660$ 
[13] Qi C R, Yi L, Su H, et al. PointNet++: deep hierarchical feature learning on point sets in a metric space[C] //Proceedings of the 31st Annual Conference on Neural Information Processing Systems. La Jolla: Neural Information Processing Systems Press, 2017: 5099-5108

[14] Lawrence S, Giles C L, Tsoi A C, et al. Face recognition: a convolutional neural-network approach[J]. IEEE Transactions on Neural Networks, 1997, 8(1): 98-113

[15] Masci J, Meier U, Cirean D, et al. Stacked convolutional auto-encoders for hierarchical feature extraction[C] //Proceedings of the 21st International Conference on Artificial Neural Networks. Heidelberg: Springer, 2011: 52-59

[16] Tschannen M, Bachem O, Lucic M. Recent advances in autoencoder-based representation learning[OL]. [2020-11-28]. https://arxiv.org/abs/1812.05069

[17] Goodfellow I, Pouget-Abadie J, Mirza M, et al. Generative adversarial networks[J]. Communications of the ACM, 2020, 63(11): 139-144

[18] Li R H, Li X Z, Fu C W, et al. PU-GAN: a point cloud upsampling adversarial network[C] //Proceedings of the IEEE/CVF International Conference on Computer Vision. Los Alamitos: IEEE Computer Society Press, 2019: 7202-7211

[19] Huang C, Li R H, Li X Z, et al. Non-local part-aware point cloud denoising[OL]. [2020-11-28]. https://arxiv.org/abs/2003. 06631

[20] Yu L Q, Li X Z, Fu C W, et al. PU-Net: point cloud upsampling network[C] //Proceedings of the 31st IEEE/CVF Conference on Computer Vision and Pattern Recognition. Los Alamitos: IEEE Computer Society Press, 2018: 2790-2799

[21] Wang Y F, Wu S H, Huang $\mathrm{H}$, et al. Patch-based progressive 3D point set upsampling[C] //Proceedings of the IEEE Conference on Computer Vision and Pattern Recognition. Los Alamitos: IEEE Computer Society Press, 2019: 5951-5960

[22] Zhang W T, Jiang H L, Yang Z S H, et al. Data-driven upsampling of point clouds[J]. Computer-Aided Design, 2019, 112: $1-13$

[23] Li C L, Cui Z, Zheng W M, et al. Action-attending graphic neural network[J]. IEEE Transactions on Image Processing, 2018, 27(7): 3657-3670

[24] Kipf $\mathrm{T}$ N, Welling $M$. Semi-supervised classification with graph convolutional networks[OL]. [2020-11-28]. https:// arxiv.org/abs/1609.02907

[25] Li G H, Muller M, Thabet A, et al. DeepGCNs: can GCNs go as deep as CNNs?[C] //Proceedings of the IEEE International Conference on Computer Vision. Los Alamitos: IEEE Computer Society Press, 2019: 9266-9275

[26] Ledig C, Theis L, Huszár F, et al. Photo-realistic single image super-resolution using a generative adversarial network[C] //Proceedings of the IEEE Conference on Computer Vision and Pattern Recognition. Los Alamitos: IEEE Computer Society Press, 2017: 105-114

[27] Isola $\mathrm{P}$, Zhu J Y, Zhou T H, et al. Image-to-image translation with conditional adversarial networks[C] //Proceedings of the
IEEE Conference on Computer Vision and Pattern Recognition. Los Alamitos: IEEE Computer Society Press, 2017: 5967-5976

[28] Chen X L, Chen B Q, Mitra N J. Unpaired point cloud completion on real scans using adversarial training[OL]. [2020-11-28]. https://arxiv.org/abs/1904.00069

[29] Yang Y Q, Feng C, Shen Y R, et al. FoldingNet: point cloud auto-encoder via deep grid deformation[C] //Proceedings of the IEEE Conference on Computer Vision and Pattern Recognition. Los Alamitos: IEEE Computer Society Press, 2018: 206-215

[30] Huang H H, Chen H L, Li J X. Deep neural network for 3D point cloud completion with multistage loss function[C] // Proceedings of the 31st Chinese Control and Decision Conference. Los Alamitos: IEEE Computer Society Press, 2019: 4604-4609

[31] Zhang W X, Long C J, Yan Q G, et al. Multi-stage point completion network with critical set supervision[J]. Computer Aided Geometric Design, 2020, 82: 101925

[32] Tchapmi L P, Kosaraju V, Rezatofighi S H, et al. TopNet: structural point cloud decoder[C] //Proceedings of the IEEE Conference on Computer Vision and Pattern Recognition. Los Alamitos: IEEE Computer Society Press, 2019: 383-392

[33] Huang Z T, Yu Y K, Xu J W, et al. PF-Net: point fractal network for 3D point cloud completion[C] //Proceedings of the IEEE Conference on Computer Vision and Pattern Recognition. Los Alamitos: IEEE Computer Society Press, 2020: 7662-7670

[34] Wang X G, Ang M H, Lee G H. Cascaded refinement network for point cloud completion[OL]. [2020-11-28]. https://arxiv. org/abs/2004.03327

[35] Zhang W X, Yan Q G, Xiao C X. Detail preserved point cloud completion via separated feature aggregation[OL]. [2020-1128]. https://arxiv.org/abs/2007.02374

[36] Wen X, Li T Y, Han Z Z, et al. Point cloud completion by skip-attention network with hierarchical folding[C] // Proceedings of the IEEE/CVF Conference on Computer Vision and Pattern Recognition. Los Alamitos: IEEE Computer Society Press, 2020: 1939-1948

[37] Pistilli F. GraphPointNet: graph convolutional neural network for point cloud denoising[D]. Turin: Polytechnic University of Turin. Department of Electronic Engineering, 2019

[38] Simonovsky M, Komodakis N. Dynamic edge-conditioned filters in convolutional neural networks on graphs[C] //Proceedings of the IEEE Conference on Computer Vision and Pattern Recognition. Los Alamitos: IEEE Computer Society Press, 2017: 29-38

[39] Duan C J, Chen S H, Kovacevic J. 3D point cloud denoising via deep neural network based local surface estimation[C] //Proceedings of the International Conference on Acoustics, Speech and Signal Processing. Los Alamitos: IEEE Computer Society Press, 2019: 8553-8557

[40] Rakotosaona M J, La Barbera V, Guerrero P, et al. PointCleanNet: learning to denoise and remove outliers from dense point clouds[J]. Computer Graphics Forum, 2020, 39(1): 185-203

[41] Yu L Q, Li X Z, Fu C W, et al. EC-Net: an edge-aware point set 
consolidation network[C] //Proceedings of the European Conference on Computer Vision. Heidelberg: Springer Press, 2018: 398-414

[42] Luo S T, Hu W. Differentiable manifold reconstruction for point cloud denoising[OL]. [2020-11-28]. https://arxiv.org/abs/2007. 13551

[43] Xu Y F, Fan T Q, Xu M Y, et al. SpiderCNN: deep learning on point sets with parameterized convolutional filters[C] //Proceedings of the European Conference on Computer Vision. Heidelberg: Springer Press, 2018: 90-105

[44] Su H, Jampani V, Sun D Q, et al. SplatNet: sparse lattice networks for point cloud processing[C] //Proceedings of the IEEE Conference on Computer Vision and Pattern Recognition. Los Alamitos: IEEE Computer Society Press, 2018: 2530-2539

[45] Li Y Y, Bu R, Sun M C, et al. PointCNN: convolution on $X$-transformed points[C] //Proceedings of the Advances in Neural Information Processing Systems. La Jolla: Neural Information Processing Systems Press, 2018: 820-830

[46] Wu W X, Qi Z A, Li F X. PointConv: deep convolutional networks on 3D point clouds[C] //Proceedings of the IEEE/CVF Conference on Computer Vision and Pattern Recognition. Los Alamitos: IEEE Computer Society Press, 2019: 9613-9622

[47] Zhao H S, Jiang L, Fu C W, et al. PointWeb: enhancing local neighborhood features for point cloud processing[C] //Proceedings of the IEEE Conference on Computer Vision and Pattern Recognition. Los Alamitos: IEEE Computer Society Press, 2019: 5550-5558

[48] Wang Y, Sun Y B, Liu Z W, et al. Dynamic graph CNN for learning on point clouds[J]. ACM Transactions on Graphics, 2019, 38(5): 1-12

[49] Jiang M Y, Wu Y R, Zhao T Q, et al. PointSIFT: a SIFT-like network module for 3D point cloud semantic segmentation[OL]. [2020-11-28]. https://arxiv.org/abs/1807.00652

[50] Cohen T S, Geiger M, Köhler J, et al. Spherical CNNs[OL]. [2020-11-28]. https://arxiv.org/abs/1801.10130

[51] Esteves C, Allen-Blanchette C, Makadia A, et al. Learning $\mathrm{SO}(3)$ equivariant representations with spherical CNNs[C] //Proceedings of the European Conference on Computer Vision. Heidelberg: Springer, 2018: 54-70

[52] You Y, Lou Y J, Liu Q, et al. Pointwise rotation-invariant network with adaptive sampling and 3D spherical voxel convolution[OL]. [2020-11-28]. https://arxiv.org/abs/1811.09361

[53] Poulenard A, Rakotosaona M J, Ponty Y, et al. Effective rotation-invariant point CNN with spherical harmonics kernels[C] //Proceedings of the International Conference on 3D Vision. Los Alamitos: IEEE Computer Society Press, 2019: 47-56

[54] Sun X, Lian Z H, Xiao J G. SRINet: learning strictly rotation-invariant representations for point cloud classification and segmentation[C] //Proceedings of the ACM International Conference on Multimedia. New York: ACM Press, 2019: 980-988

[55] Li X Z, Li R H, Chen G Y, et al. A rotation-invariant framework for deep point cloud analysis[OL]. [2020-11-28]. https:// arxiv.org/abs/2003.07238
[56] Fan H Q, Su H, Guibas L. A point set generation network for $3 \mathrm{D}$ object reconstruction from a single image[C] //Proceedings of the 30th IEEE Conference on Computer Vision and Pattern Recognition. Los Alamitos: IEEE Computer Society Press, 2017: 2463-2471

[57] Rubner Y, Tomasi C, Guibas L J. The earth mover's distance as a metric for image retrieval[J]. International Journal of Computer Vision, 2000, 40(2): 99-121

[58] Sun Y L, Miao Y W, Chen J Z, et al. PGCNet: patch graph convolutional network for point cloud segmentation of indoor scenes[J]. Visual Computer, 2020, 36(10-12): 2407-2418

[59] Te G S, Hu W, Guo Z M, et al. RGCNN: regularized graph CNN for point cloud segmentation[C] //Proceedings of the 26th ACM International Conference on Multimedia. New York: ACM Press, 2018: 746-754

[60] Wang X L, Girshick R, Gupta A, et al. Non-local neural networks[C] //Proceedings of the IEEE Conference on Computer Vision and Pattern Recognition. Los Alamitos: IEEE Computer Society Press, 2018: 7794-7803

[61] Chang A X, Funkhouser T, Guibas L, et al. ShapeNet: an information-rich 3D model repository[OL]. [2020-11-28]. https://arxiv.org/abs/1512.03012

[62] Dai A, Chang A X, Savva M, et al. ScanNet: richly-annotated $3 D$ reconstructions of indoor scenes[C] //Proceedings of the IEEE Conference on Computer Vision and Pattern Recognition. Los Alamitos: IEEE Computer Society Press, 2017: 2432-2443

[63] Geiger A, Lenz P, Stiller C, et al. Vision meets robotics: the KITTI dataset[J]. The International Journal of Robotics Research, 2013, 32(11): 1231-1237

[64] Wu Z R, Song S R, Khosla A, et al. 3D ShapeNets: a deep representation for volumetric shapes[C] //Proceedings of the IEEE Conference on Computer Vision and Pattern Recognition. Los Alamitos: IEEE Computer Society Press, 2015: 1912-1920

[65] Serna A, Marcotegui B, Goulette F, et al. Paris-rue-Madame database: a 3D mobile laser scanner dataset for benchmarking urban detection, segmentation and classification methods[C] //Proceedings of the 4th International Conference on Pattern Recognition, Applications and Methods. Setúbal: SciTePress, 2014: $1-6$

[66] Lian Z, Zhang J, Choi S, et al. Non-rigid 3D shape retrieval[C] //Proceedings of the Eurographics Workshop on 3D Object Retrieval. New York: ACM Press, 2015: 107-120

[67] Chang A, Dai A, Funkhouser T, et al. Matterport3D: learning from RGB-D data in indoor environments[C] //Proceedings of International Conference on 3D Vision. Los Alamitos: IEEE Computer Society Press, 2017: 667-676

[68] Zeng J, Cheung G N, Ng M, et al. 3D point cloud denoising using graph laplacian regularization of a low dimensional manifold model[J]. IEEE Transactions on Image Processing, 2019, 29: 3474-3489

[69] Tagliasacchi A, Zhang H, Cohen-Or D. Curve skeleton extraction from incomplete point cloud[C] //Proceedings of ACM SIGGRAPH Conference, New York: ACM Press, 2009: Article 
No.71

[70] Chen S H, Duan C J, Yang Y Q, et al. Deep unsupervised learning of 3D point clouds via graph topology inference and filtering[J]. IEEE Transactions on Image Processing, 2019, 29: 3183-3198

[71] Alexa M, Behr J, Cohen-Or D, et al. Computing and rendering point set surfaces[J]. IEEE Transactions on Visualization and Computer Graphics, 2003, 9(1): 3-15

[72] Sarkar K, Varanasi K, Stricker D. Learning quadrangulated patches for 3D shape parameterization and completion[C] //Proceedings of International Conference on 3D Vision. Los Alamitos: IEEE Computer Society Press, 2017: 383-392

[73] Lu X Q, Wu S H, Chen H H, et al. GPF: GMM-inspired feature-preserving point set filtering[J]. IEEE Transactions on Visualization and Computer Graphics, 2018, 24(8): 2315-2326

[74] Qian Y, Hou J H, Kwong S, et al. PUGeo-Net: a geome- try-centric network for 3D point cloud upsampling[OL]. [2020-11-28]. https://arxiv.org/abs/2002.10277

[75] He Jun, Zhang Caiqing, Li Xiaozhen, et al. Survey of research on multimodal fusion technology for deep learning[J]. Computer Engineering, 2020, 46(5): 1-11(in Chinese)

(何俊, 张彩庆, 李小珍, 等. 面向深度学习的多模态融合技 术研究综述 [J]. 计算机工程, 2020, 46(5): 1-11)

[76] Zhang Y D, Funkhouser T. Deep depth completion of a single RGB-D image[C] //Proceedings of the 31st IEEE/CVF Conference on Computer Vision and Pattern Recognition. Los Alamitos: IEEE Computer Society Press, 2018: 175-185

[77] Qiu J X, Cui Z P, Zhang Y D, et al. DeepLiDAR: deep surface normal guided depth prediction for outdoor scene from sparse lidar data and single color image[C] //Proceedings of the IEEE Conference on Computer Vision and Pattern Recognition. Los Alamitos: IEEE Computer Society Press, 2019: 3313-3322 